\title{
EXISTENCE AND CONCENTRATE BEHAVIOR OF SCHRÖDINGER EQUATIONS WITH CRITICAL EXPONENTIAL GROWTH IN $\mathbb{R}^{N}$
}

\author{
JiAN ZHANG - WENMING ZoU
}

Abstract. We consider the nonlinear Schrödinger equation

$$
-\Delta u+(1+\mu g(x)) u=f(u) \quad \text { in } \mathbb{R}^{N},
$$

where $N \geq 3, \mu \geq 0$; the function $g \geq 0$ has a potential well and $f$ has critical growth. By using variational methods, the existence and concentration behavior of the ground state solution are obtained.

\section{Introduction}

In this paper, we are concerned with the following Schrödinger equation:

$$
-\Delta u+(1+\mu g(x)) u=f(u) \quad \text { in } \mathbb{R}^{N},
$$

where $N \geq 3, \mu \geq 0$, the potential $g$ is nonnegative and the nonlinear term $f$ is of critical growth. This equation arises in many models of mathematical physics and has been studied under various assumptions imposed on $\mu, g$ and $f$.

Recall that $u$ is a ground state solution of (1.1) if and only if $u$ solves (1.1) and minimizes the functional associated to (1.1) among all possible nontrivial

2010 Mathematics Subject Classification. 35J60, 35A15.

Key words and phrases. Schrödinger equations; critical growth; ground state solution; concentration.

Jian Zhang is supported by NSFC (11401583) and the Fundamental Research Funds for the central universities (16CX02051A).

Wenming Zou is supported by NSFC $(11025106,11371212,11271386)$ and the Both-Side Tsinghua Fund. 
solutions. When $\mu=0$ and $f$ is a subcritical function, almost necessary and sufficient conditions for the existence of ground state solutions to (1.1) are given by Berestycki and Lions in [9] when $N \geq 3$ and Berestycki et al. in [8] for $N=2$. Subsequently, the authors in [1], [40] attempted to complete the study initiated in [8], [9], by considering the nonlinearities with critical growth. The main difficulty related to (1.1) is the lack of compactness. Several approaches have been developed to overcome this difficulty. See for example [11], [23], [24], [27], [31] for the subcritical cases and [39] for the critical cases. When $\mu>0$, many authors have worked on equation (1.1) in various forms and obtained numerous results on the existence, multiplicity and concentration behavior of solutions. In particular, in [7], Bartsch and Wang considered the subcritical problem

$$
-\Delta u+(1+\mu g(x)) u=u^{p-1} \quad \text { in } \mathbb{R}^{N},
$$

where $N \geq 3,2<p<2^{*}:=2 N /(N-2)$ and the function $g$ satisfies the following conditions:

$\left(\mathrm{g}_{1}\right) g \in \mathbb{C}\left(\mathbb{R}^{N}, \mathbb{R}\right), g \geq 0$.

( $\left.\mathrm{g}_{2}\right) \quad \Omega:=\operatorname{int} g^{-1}(0)$ is non-empty and has smooth boundary and $\bar{\Omega}=g^{-1}(0)$.

$\left(\mathrm{g}_{3}\right)$ There exists $M_{0}>0$ such that meas $\left\{x \in \mathbb{R}^{N}: g(x) \leq M_{0}\right\}<\infty$, where meas denotes the Lebesgue measure on $\mathbb{R}^{N}$.

Under the above assumptions, they showed that for $\mu$ large enough, problem (1.2) admits a positive ground state solution. Moreover, the ground state solution converges (as $\mu \rightarrow \infty$ ) to a positive ground state solution of the following limit equation:

$$
-\Delta u+u=u^{p-1}, \quad u \in H_{0}^{1}(\Omega) .
$$

Multiplicity of solutions for (1.3) were also considered. It is remarkable that the function $1+\mu g$ represents a potential well whose depth is controlled by $\mu$ and, when $\mu \rightarrow \infty$, a certain of concentration behavior occurs. When the number of components contained in $\Omega$ is more than one, we refer the reader to [19] for multiplicity of positive solutions and to [32] for multiplicity of positive and signchanging solutions. For other related results, see [5], [6], [26], [34]-[35] and the references therein.

However, in all papers mentioned above the nonlinearities are assumed to be subcritical. Naturally, it is interesting to ask what happens when the nonlinearity is of critical growth? We remark that Clapp and Ding [12] investigated the following problem:

$$
-\Delta u+\mu g(x) u=\lambda u+u^{2^{*}-1} \quad \text { in } \mathbb{R}^{N},
$$

where $N \geq 4, \lambda>0$ and $g$ satisfies $\left(\mathrm{g}_{1}\right)-\left(\mathrm{g}_{3}\right)$ with $\Omega$ bounded. For $\lambda$ small and $\mu$ large, the existence and multiplicity of solutions for (1.4) were obtained and 
a concentration behavior was observed as $\mu \rightarrow \infty$. See also [13], [14] for other critical cases.

The main goal of this paper is to study another class of nonlinearities with critical growth and obtain the existence and concentration behavior of the positive ground state to (1.1). We introduce the following hypotheses on $g$ and $f$ :

$\left(\mathrm{G}_{1}\right) g \in \mathbb{C}\left(\mathbb{R}^{N}, \mathbb{R}\right)$ and $g(x) \geq 0$ for all $x \in \mathbb{R}^{N}$;

$\left(\mathrm{G}_{2}\right)$ the set $\Omega_{0}:=\left\{x \in \mathbb{R}^{N}: g(x)=0\right\}$ is bounded and has non-empty interior;

$\left(\mathrm{G}_{3}\right)$ there exists $g_{0}>0$ such that the set $\left\{x \in \mathbb{R}^{N}: g(x) \leq g_{0}\right\}$ is bounded;

$\left(\mathrm{f}_{1}\right) f \in \mathbb{C}\left(\mathbb{R}^{+}, \mathbb{R}\right)$

$\left(\mathrm{f}_{2}\right) \lim _{t \rightarrow 0^{+}} f(t) / t=0$;

$\left(\mathrm{f}_{3}\right) \lim _{t \rightarrow+\infty} f(t) /\left(t^{2^{*}-1}\right)=K>0$;

$\left(\mathrm{f}_{4}\right)$ there exists $\theta \in\left(2,2^{*}\right]$ such that $f(t) t-\theta F(t) \geq 0$ for $t \geq 0$, where $F(t)=\int_{0}^{t} f(s) d s$

$\left(\mathrm{f}_{5}\right)$ there exist $q \in\left(2,2^{*}\right)$ and $\lambda>0$ such that $f(t) \geq \lambda t^{q-1}$ for $t \geq 0$.

Before stating the main results of the current paper, we need some definitions. Denote the best Sobolev constants by

$$
S:=\inf _{u \in D^{1,2}\left(\mathbb{R}^{N}\right) \backslash\{0\}} \frac{\int_{\mathbb{R}^{N}}|\nabla u|^{2} d x}{\left(\int_{\mathbb{R}^{N}}|u|^{2^{*}} d x\right)^{2 / 2^{*}}}
$$

and

$$
\begin{gathered}
S_{q}:=\inf _{u \in H_{0}^{1}\left(\Omega_{0}\right) \backslash\{0\}} \frac{\int_{\Omega_{0}}\left(|\nabla u|^{2}+u^{2}\right) d x}{\left(\int_{\Omega_{0}}|u|^{q} d x\right)^{2 / q}}, \\
C_{q}:=\inf _{u \in H^{1}\left(\mathbb{R}^{N}\right) \backslash\{0\}} \frac{\int_{\mathbb{R}^{N}}\left(|\nabla u|^{2}+|u|^{2}\right) d x}{\left(\int_{\mathbb{R}^{N}}|u|^{q} d x\right)^{2 / q}} .
\end{gathered}
$$

We distinguish two different situations. Firstly, we consider the case of $\mu>0$.

Theorem 1.1. Assume that $\left(\mathrm{G}_{1}\right)-\left(\mathrm{G}_{3}\right)$ and $\left(\mathrm{f}_{1}\right)-\left(\mathrm{f}_{5}\right)$ hold with

$$
\lambda>\left[\frac{2 \theta(q-2)}{q(\theta-2)}\right]^{(q-2) / 2}\left[\frac{8(N-1)}{N-2}\right]^{(q-2)(N-2) / 4} \frac{S_{q}^{q / 2}}{S^{N(q-2) / 4}} .
$$

Then there exists $\mu_{0}>0$ such that for $\mu>\mu_{0}$, problem (1.1) admits a positive ground state solution. 
REMARK 1.2. Condition $\left(f_{3}\right)$ characterizes equation (1.1) to be the critical growth case. A typical example satisfying $\left(f_{1}\right)-\left(f_{5}\right)$ is the function

$$
f(t)=\lambda t^{q-1}+K t^{2^{*}-1}, \quad t \geq 0 .
$$

For this case, we may choose $\theta=q \in\left(2,2^{*}\right)$.

TheOREM 1.3. For $\mu_{n}>\mu_{0}$, let $u_{\mu_{n}}$ be a sequence of positive ground state solutions from Theorem 1.1 with $\mu_{n} \rightarrow \infty$. Then up to a subsequence, $u_{\mu_{n}} \rightarrow u$ in $H^{1}\left(\mathbb{R}^{N}\right)$ as $\mu_{n} \rightarrow+\infty$, where $u(x)=0$ a.e. $x \in \mathbb{R}^{N} \backslash \Omega_{0}$. Moreover, if $\partial \Omega_{0}$ is smooth, then $u$ is a positive solution to the following problem:

$$
-\Delta u+u=f(u), \quad u \in H_{0}^{1}\left(\Omega_{0}\right) .
$$

Similar conditions to $\left(\mathrm{G}_{1}\right)-\left(\mathrm{G}_{3}\right)$ were introduced in [6], [7]. However, the methods of [6], [7] do not apply to (1.1) due to the critical exponential growth. In fact, it is known that the embedding is not compact even if the domain is bounded. In [12], the authors considered a critical case. Unfortunately, it seems that the device used in [12] does not work for (1.1). In this paper, we shall apply a penalization approach developed by del Pino and Felmer [15]. Such an approach has been widely used in dealing with singularly perturbed problems.

On the other hand, lots of papers have been devoted to studying the existence and concentration phenomenon of solutions to the following singularly perturbed problem:

$$
-\varepsilon^{2} \Delta u+V(x) u=f(u) \quad \text { in } \mathbb{R}^{N}
$$

with various hypotheses on $V$ and $f$. Denoting $v(x)=u(\varepsilon x)$, equation (1.6) is reduced to

$$
-\Delta v+V(\varepsilon x) v=f(v) \quad \text { in } \mathbb{R}^{N} .
$$

The existence of a single spike solution which concentrates around any given non-degenerate critical point of the potential $V$ was first constructed in [20] for $N=1$ and $f(u)=u^{3}$. Later on, Oh [29], [30] extended this result to higher dimension cases with $f(u)=|u|^{p-1} u, 1<p<(N+2) /(N-2)$. He also considered multiple spike solutions. The arguments in [20], [29], [30] are based on the Lyapunov-Schmidt reduction and the uniqueness and non-degeneracy of the ground state to the limiting equation

$$
-\Delta v+V(0) v=f(v) \text { in } \mathbb{R}^{N} .
$$

Reduction methods were also found suitable for finding solutions of (1.7) concentrating around possibly degenerate critical points of $V$. See for example [3], [28] and the references therein. However, the uniqueness and non-degeneracy of the ground state solution are usually rather difficult to check. To overcome this 
difficulty, the variational approach by Rabinowitz [31] is proved to be successful. Later on, by introducing a penalization approach, del Pino and Felmer [15] proved a localized version of results in [31]. Jeanjean and Tanaka [25] extended the work of [15] to a more general superlinear nonlinearity. The asymptotically linear case was also considered in [25]. See [4], [10], [16]-[18], [21] for the subcritical cases and [38] for the critical cases. We note that solutions of (1.7) concentrate at a solution of the limit equation (1.8). This concentration behaviors are rather different from that of solutions to (1.1).

Now we consider the case of $\mu=0$ in (1.1). Our aim is to improve the result obtained in [1]. Recall that in [1], the authors proved the existence of the ground state solution to (1.1) under assumptions $\left(f_{1}\right)-\left(f_{5}\right)$ with $\mu=0, \theta=2$ and

$$
\lambda>\left[2^{(2-N) / 2} S^{-N / 2} N\left(\frac{2 N}{N-2}\right)^{(N-2) / 2}\right]^{(q-2) / 2}\left[\frac{q-2}{2 q}\right]^{(q-2) / 2} C_{q}^{q / 2} .
$$

Comparing with the result of [1], our argument is very simple. In particular, we can remove condition $\left(f_{4}\right)$ by applying an indirect method developed in [22].

TheOREm 1.4. Assume $\mu=0$. Suppose that $\left(\mathrm{f}_{1}\right)-\left(\mathrm{f}_{3}\right)$ and $\left(\mathrm{f}_{5}\right)$ hold with

$$
\lambda>\left(\frac{q-2}{2 q}\right)^{(q-2) / 2}\left(N S^{-N / 2}\right)^{(q-2) / 2} C_{q}^{q / 2} .
$$

Then problem (1.1) admits a positive ground state solution $u^{\lambda}$. Moreover,

$$
\lim _{\lambda \rightarrow \infty} \int_{\mathbb{R}^{N}}\left(\left|\nabla u^{\lambda}\right|^{2}+\left|u^{\lambda}\right|^{2}-F\left(u^{\lambda}\right)\right) d x=0 .
$$

REMARK 1.5. When replacing $\left(f_{5}\right)$ in Theorem 1.4 by the following condition:

$\left(\mathrm{f}_{6}\right)$ there exist $D>0$ and $q \in\left(2,2^{*}\right)$ such that $f(t) \geq D t^{q-1}+K t^{2^{*}-1}$ for $t \geq 0$,

the authors in [40] proved that problem (1.1) also admits a positive ground state solution for $N=3$ with $q>4$, or $N \geq 4$. The main idea in obtaining this result is in trying to solve the constraint minimization problem corresponding to (1.1). It is remarkable that $\left(f_{5}\right)$ is different from $\left(f_{6}\right)$. In fact, when fixing $\lambda>((q-2) / 2 q)^{(q-2) / 2}\left(N S^{-N / 2}\right)^{(q-2) / 2} C_{q}^{q / 2}$, we can define a function satisfying $\left(\mathrm{f}_{1}\right)-\left(\mathrm{f}_{3}\right)$ and $\left(\mathrm{f}_{5}\right)$ by $f(t)=\max \left\{\lambda t^{q-1}, K t^{2^{*}-1}\right\}$, for $t \geq 0$. Clearly for this case the function $f$ does not satisfy $\left(\mathrm{f}_{6}\right)$.

The outline of this paper is as follows: in Section 2, we establish some important lemmas. In Section 3, we prove Theorems 1.1-1.3. In Section 4, we prove Theorem 1.4.

\section{Notations.}

- $C$ denotes a positive (possibly different) constant. 
- $B_{r}\left(x_{0}\right)$ denotes the open ball centered at $x_{0}$ with radius $r>0,\left|B_{r}\left(x_{0}\right)\right|$ denotes the volume of $B_{r}\left(x_{0}\right)$.

- For $2 \leq s \leq \infty,\|u\|_{s}$ denotes the usual norm of $L^{s}\left(\mathbb{R}^{N}\right),\|u\|_{L^{s}\left(B_{r}\left(x_{0}\right)\right)}$ denotes the usual norm of $L^{s}\left(B_{r}\left(x_{0}\right)\right)$.

- $H^{1}\left(\mathbb{R}^{N}\right)$ denotes the Hilbert space equipped with the norm

$$
\|u\|_{H^{1}}^{2}:=\int_{\mathbb{R}^{N}}\left(|\nabla u|^{2}+|u|^{2}\right) d x
$$

\section{Preliminary lemmas}

In this section, we assume that the hypotheses of Theorem 1.1 hold. Let

$$
E:=\left\{u \in H^{1}\left(\mathbb{R}^{N}\right): \int_{\mathbb{R}^{N}}(1+\mu g(x)) u^{2} d x<\infty\right\}
$$

be a subspace of $H^{1}\left(\mathbb{R}^{N}\right)$ equipped with the norm

$$
\|u\|_{\mu}^{2}:=\int_{\mathbb{R}^{N}}\left(|\nabla u|^{2}+(1+\mu g(x)) u^{2}\right) d x .
$$

From $\left(\mathrm{G}_{1}\right)$, we know that the embedding $E \hookrightarrow H^{1}\left(\mathbb{R}^{N}\right)$ is continuous.

As we look for positive solutions, without loss of generality, we may assume $f(t)=0$ for $t \leq 0$. Then from the maximum principle, any nontrivial solution of (1.1) will be positive. Define the functional $I_{\mu}$ on $E$ by

$$
I_{\mu}(u)=\frac{1}{2}\|u\|_{\mu}^{2}-\int_{\mathbb{R}^{N}} F(u) d x, \quad u \in E .
$$

It is easy to check that the functional $I_{\mu}: E \mapsto \mathbb{R}$ is of class $\mathbb{C}^{1}$. Moreover, the critical points of $I_{\mu}$ are the weak solutions to (1.1). For the simplicity, we may assume that $K=1$. Set $h(t)=f(t)-\left(t^{+}\right)^{2^{*}-1}$. Then

$$
I_{\mu}(u)=\frac{1}{2}\|u\|_{\mu}^{2}-\int_{\mathbb{R}^{N}} H(u) d x-\frac{1}{2^{*}} \int_{\mathbb{R}^{N}}\left|u^{+}\right|^{2^{*}} d x,
$$

where $u \in E, H(u)=\int_{0}^{u} h(t) d t$. Instead of dealing with $I_{\mu}(u)$ directly, we will consider a truncated problem first. Similarly to [15], we modify the nonlinearity $f$. By $\left(\mathrm{G}_{3}\right)$, we can find $R>0$ such that $\Omega_{0} \subset B_{R}(0)$ and $g(x) \geq g_{0}$ for $|x| \geq R$. For $\kappa>2$, we define $\widetilde{f}(t)=\min \left\{f(t),\left(1+g_{0} \mu\right) t^{+} / \kappa\right\}$. Note that we can choose $\chi \in \mathbb{C}\left(\mathbb{R}^{N}, \mathbb{R}\right)$ such that $\chi(x)=1$ for $|x| \leq R, \chi(x)=0$ for $|x| \geq R+1$ and $0 \leq \chi(x) \leq 1$. Then we define $k(x, t)=\chi(x) f(t)+(1-\chi(x)) \widetilde{f}(t)$. Consider the truncated functional $J_{\mu}$ on $E$ defined by

$$
J_{\mu}(u)=\frac{1}{2}\|u\|_{\mu}^{2}-\int_{\mathbb{R}^{N}} K(x, u) d x
$$

where

$K(x, u)=\int_{0}^{u} k(x, s) d s=\chi(x) F(u)+(1-\chi(x)) \widetilde{F}(u) \quad$ and $\quad \widetilde{F}(u)=\int_{0}^{u} \widetilde{f}(s) d s$. 
Now we try to find a critical point $u_{\mu}$ of $J_{\mu}$ on $E$ via a mountain pass argument and investigate properties of $u_{\mu}$. We will show that for $\mu>0$ large, $u_{\mu}$ also solves the original problem (1.1).

Lemma 2.1. For $\mu>0$, there is a sequence $\left\{u_{n}\right\} \subset E$ such that $\left\{u_{n}\right\}$ is bounded in $E$,

$$
J_{\mu}\left(u_{n}\right) \rightarrow c_{\mu} \in\left(0, \frac{\theta-2}{4 \theta}\left[\frac{N-2}{8(N-1)}\right]^{(N-2) / 2} S^{N / 2}\right) \quad \text { and } \quad J_{\mu}^{\prime}\left(u_{n}\right) \rightarrow 0 .
$$

Proof. Conditions $\left(\mathrm{f}_{1}\right)-\left(\mathrm{f}_{3}\right)$ imply that for all $\varepsilon>0$ there exists $C(\varepsilon)>0$ such that

$$
|f(u)| \leq \varepsilon|u|+C(\varepsilon)|u|^{2^{*}-1} .
$$

Note that

$$
\int_{\mathbb{R}^{N}} K(x, u) d x \leq \int_{\mathbb{R}^{N}} F(u) d x
$$

Together with $\left(G_{1}\right),(2.3)$ and the Sobolev embedding theorem, we can find $r>0$ such that $J_{\mu}(u) \geq c>0$ for $\|u\|_{\mu}=r$. Condition $\left(f_{5}\right)$ implies that $F(u) \geq \lambda\left|u^{+}\right|^{q} / q$. Choose $\varphi \in C_{0}^{\infty}\left(\Omega_{0}\right)$ such that $\varphi \geq 0$ in $\Omega_{0}$ and $\varphi \neq 0$. Then we have $\lim _{t \rightarrow+\infty} J_{\mu}(t \varphi)=-\infty$. Define

$$
c_{\mu}=\inf _{\gamma \in \Gamma} \max _{t \in[0,1]} J_{\mu}(\gamma(t)),
$$

where $\Gamma=\left\{\gamma \in C([0,1], E): \gamma(0)=0, J_{\mu}(\gamma(1))<0\right\}$. It follows from the mountain pass theorem in [2] that there is a sequence $\left\{u_{n}\right\} \subset E$ such that $J_{\mu}\left(u_{n}\right) \rightarrow c_{\mu} \geq c$ and $J_{\mu}^{\prime}\left(u_{n}\right) \rightarrow 0$.

It is well-known that $S_{q}$ is attained. Then we can find $\psi \in H_{0}^{1}\left(\Omega_{0}\right)$ such that $\psi \geq 0$ in $\Omega_{0}, \psi \neq 0$ and

$$
S_{q}=\frac{\int_{\Omega_{0}}\left(|\nabla \psi|^{2}+\psi^{2}\right) d x}{\left(\int_{\Omega_{0}}|\psi|^{q} d x\right)^{2 / q}} .
$$

From the definition of $c_{\mu}$, it can be derived that $c_{\mu} \leq \sup _{t \geq 0} J_{\mu}(t \psi)=\sup _{t \geq 0} I_{\mu}(t \psi)$. From $\left(G_{2}\right)$ and $\left(f_{5}\right)$,

$$
\begin{aligned}
\sup _{t \geq 0} I_{\mu}(t \psi) & \leq \sup _{t \geq 0}\left[\frac{1}{2} t^{2} \int_{\Omega_{0}}\left(|\nabla \psi|^{2}+\psi^{2}\right) d x-\frac{\lambda}{q} t^{q} \int_{\Omega_{0}}|\psi|^{q} d x\right] \\
& =\left(\frac{1}{2}-\frac{1}{q}\right) \frac{1}{\lambda^{2 /(q-2)}} S_{q}^{q /(q-2)}<\frac{\theta-2}{4 \theta}\left[\frac{N-2}{8(N-1)}\right]^{(N-2) / 2} S^{N / 2} .
\end{aligned}
$$

Then we have

$$
c_{\mu}<\frac{\theta-2}{4 \theta}\left[\frac{N-2}{8(N-1)}\right]^{(N-2) / 2} S^{N / 2} .
$$


On the other hand, by $\left(\mathrm{f}_{4}\right)$,

$$
\begin{aligned}
c_{\mu}+o_{n} & (1)+o_{n}(1)\left\|u_{n}\right\|_{\mu}=J_{\mu}\left(u_{n}\right)-\frac{1}{\theta}\left(J_{\mu}^{\prime}\left(u_{n}\right), u_{n}\right) \\
& \geq\left(\frac{1}{2}-\frac{1}{\theta}\right)\left\|u_{n}\right\|_{\mu}^{2}+\int_{|x| \geq R}(1-\chi(x))\left[\frac{1}{\theta} \widetilde{f}\left(u_{n}\right) u_{n}-\widetilde{F}\left(u_{n}\right)\right] d x \\
& \geq\left(\frac{1}{2}-\frac{1}{\theta}\right)\left\|u_{n}\right\|_{\mu}^{2}-\left(\frac{1}{2}-\frac{1}{\theta}\right) \frac{1}{\kappa} \int_{|x| \geq R}\left(1+\mu g_{0}\right) u_{n}^{2} d x \\
& \geq\left(\frac{1}{2}-\frac{1}{\theta}\right)\left\|u_{n}\right\|_{\mu}^{2}-\left(\frac{1}{2}-\frac{1}{\theta}\right) \frac{1}{\kappa} \int_{\mathbb{R}^{N}}(1+\mu g(x)) u_{n}^{2} d x \\
& \geq \frac{\theta-2}{4 \theta}\left\|u_{n}\right\|_{\mu}^{2},
\end{aligned}
$$

which implies that $\left\|u_{n}\right\|_{\mu}$ is bounded in $E$.

Now we investigate properties of the sequence $\left\{u_{n}\right\}$ obtained in Lemma 2.1.

LEMmA 2.2. There is a sequence $\left\{z_{n}\right\} \subset \mathbb{R}^{N}$ and $\beta>0$ such that

$$
\int_{B_{1}\left(z_{n}\right)} u_{n}^{2} d x \geq \beta
$$

Moreover, the sequence $\left\{z_{n}\right\}$ is bounded in $\mathbb{R}^{N}$.

Proof. We may assume that $u_{n} \geq 0$ in $E$. Now we prove the first result. Assume to the contrary that

$$
\lim _{n \rightarrow \infty} \sup _{z \in \mathbb{R}^{N}} \int_{B_{1}(z)} u_{n}^{2} d x=0 .
$$

By the Lions lemma in [37], we obtain

$$
u_{n} \rightarrow 0 \text { in } L^{t}\left(\mathbb{R}^{N}\right) \text {, for all } t \in\left(2,2^{*}\right) .
$$

Note that

$$
\int_{\mathbb{R}^{N}} k\left(x, u_{n}\right) u_{n} d x \leq \int_{\mathbb{R}^{N}} f\left(u_{n}\right) u_{n} d x
$$

hence

$$
o_{n}(1)=\left(J_{\mu}^{\prime}\left(u_{n}\right), u_{n}\right) \geq\left\|u_{n}\right\|_{\mu}^{2}-\int_{\mathbb{R}^{N}} h\left(u_{n}\right) u_{n} d x-\int_{\mathbb{R}^{N}}\left|u_{n}\right|^{2^{*}} d x .
$$

By $\left(\mathrm{f}_{1}\right)-\left(\mathrm{f}_{3}\right)$, there exists $s \in\left(2,2^{*}\right)$ such that for all $\varepsilon>0$, there exists $C(\varepsilon)>0$ satisfying $\left|h\left(u_{n}\right) u_{n}\right| \leq \varepsilon\left|u_{n}\right|^{2}+\varepsilon\left|u_{n}\right|^{2^{*}}+C(\varepsilon)\left|u_{n}\right|^{s}$. Together with (2.5), we obtain

$$
\int_{\mathbb{R}^{N}} h\left(u_{n}\right) u_{n} d x=o_{n}(1) .
$$

Thus,

$$
o_{n}(1) \geq\left\|u_{n}\right\|_{\mu}^{2}-\int_{\mathbb{R}^{N}}\left|u_{n}\right|^{2^{*}} d x
$$


On the other hand,

$$
\begin{aligned}
c_{\mu}+o_{n}(1) & =J_{\mu}\left(u_{n}\right)-\frac{1}{2^{*}}\left(J_{\mu}^{\prime}\left(u_{n}\right), u_{n}\right) \\
& =\frac{1}{N}\left\|u_{n}\right\|_{\mu}^{2}+\int_{\mathbb{R}^{N}}\left[\frac{1}{2^{*}} k\left(x, u_{n}\right) u_{n}-K\left(x, u_{n}\right)\right] d x .
\end{aligned}
$$

Observe that

$$
\begin{aligned}
\int_{\mathbb{R}^{N}} & {\left[\frac{1}{2^{*}} k\left(x, u_{n}\right) u_{n}-K\left(x, u_{n}\right)\right] d x } \\
= & \int_{|x| \leq R}\left[\frac{1}{2^{*}} f\left(u_{n}\right) u_{n}-F\left(u_{n}\right)\right] d x+\int_{|x|>R} \chi(x)\left[\frac{1}{2^{*}} f\left(u_{n}\right) u_{n}-F\left(u_{n}\right)\right] d x \\
& +\int_{|x|>R}(1-\chi(x))\left[\frac{1}{2^{*}} \tilde{f}\left(u_{n}\right) u_{n}-\widetilde{F}\left(u_{n}\right)\right] d x \\
= & \int_{|x| \leq R}\left[\frac{1}{2^{*}} h\left(u_{n}\right) u_{n}-H\left(u_{n}\right)\right] d x+\int_{|x|>R} \chi(x)\left[\frac{1}{2^{*}} h\left(u_{n}\right) u_{n}-H\left(u_{n}\right)\right] d x \\
& +\int_{|x|>R}(1-\chi(x))\left[\frac{1}{2^{*}} \widetilde{f}\left(u_{n}\right) u_{n}-\widetilde{F}\left(u_{n}\right)\right] d x .
\end{aligned}
$$

Similarly to (2.6), we also have $\int_{\mathbb{R}^{N}} H\left(u_{n}\right) d x=o_{n}(1)$. Then

$$
\begin{aligned}
\int_{\mathbb{R}^{N}}\left[\frac{1}{2^{*}} k\left(x, u_{n}\right) u_{n}-\right. & \left.K\left(x, u_{n}\right)\right] d x \\
& =\int_{|x|>R}(1-\chi(x))\left[\frac{1}{2^{*}} \tilde{f}\left(u_{n}\right) u_{n}-\widetilde{F}\left(u_{n}\right)\right] d x+o_{n}(1) .
\end{aligned}
$$

Therefore,

$$
\begin{aligned}
c_{\mu}+o_{n}(1) & =\frac{1}{N}\left\|u_{n}\right\|_{\mu}^{2}+\int_{|x|>R}(1-\chi(x))\left[\frac{1}{2^{*}} \widetilde{f}\left(u_{n}\right) u_{n}-\widetilde{F}\left(u_{n}\right)\right] d x \\
& \geq \frac{1}{N}\left\|u_{n}\right\|_{\mu}^{2}-\frac{1}{\kappa N} \int_{\mathbb{R}^{N}}(1+\mu g(x)) u_{n}^{2} d x \\
& \geq \frac{1}{N} \int_{\mathbb{R}^{N}}\left|\nabla u_{n}\right|^{2} d x+\frac{1}{2 N} \int_{\mathbb{R}^{N}}(1+\mu g(x)) u_{n}^{2} d x .
\end{aligned}
$$

Assume that

$$
\lim _{n \rightarrow \infty}\left[\int_{\mathbb{R}^{N}}\left|\nabla u_{n}\right|^{2} d x+\frac{1}{2} \int_{\mathbb{R}^{N}}(1+\mu g(x)) u_{n}^{2} d x\right]=l .
$$

Then by (2.7), we have

$$
\lim _{n \rightarrow \infty} \int_{\mathbb{R}^{N}}\left|u_{n}\right|^{2^{*}} d x \geq l .
$$

Moreover, it follows from $c_{\mu}>0$ that $l>0$. Thus, by

$$
S \leq \frac{\int_{\mathbb{R}^{N}}\left|\nabla u_{n}\right|^{2} d x}{\left(\int_{\mathbb{R}^{N}}\left|u_{n}\right|^{2^{*}} d x\right)^{2 / 2^{*}},}
$$


we get $l \geq S^{N / 2}$. Together with (2.8), there holds $c_{\mu} \geq S^{N / 2} / N$. On the other hand, for $N \geq 3$, we have

$$
c_{\mu}<\frac{\theta-2}{4 \theta}\left[\frac{N-2}{8(N-1)}\right]^{(N-2) / 2} S^{N / 2}<\frac{2}{8^{N / 2}} S^{N / 2}<\frac{1}{N} S^{N / 2},
$$

a contradiction. Therefore, there is a sequence $\left\{z_{n}\right\} \subset \mathbb{R}^{N}$ and $\beta>0$ such that

$$
\int_{B_{1}\left(z_{n}\right)} u_{n}^{2} d x \geq \beta
$$

Now we prove that $\left\{z_{n}\right\}$ is bounded in $\mathbb{R}^{N}$. For $L>R+1$, define $\Psi_{L} \in C_{0}^{\infty}\left(\mathbb{R}^{N}\right)$ such that $\Psi_{L}(x)=0$ for $|x| \leq L, \Psi_{L}(x)=1$ for $|x| \geq 2 L$ and $0 \leq \Psi_{L}(x) \leq 1$. Moreover, $\left|\nabla \Psi_{L}\right| \leq C / L$. Note that

$$
o_{n}(1)=\int_{\mathbb{R}^{N}}\left[\nabla u_{n} \nabla\left(\Psi_{L} u_{n}\right)+(1+\mu g(x)) u_{n}^{2} \Psi_{L}\right] d x-\int_{\mathbb{R}^{N}} k\left(x, u_{n}\right) u_{n} \Psi_{L} d x,
$$

hence we have

$$
\begin{aligned}
\int_{\mathbb{R}^{N}} & {\left[\left|\nabla u_{n}\right|^{2} \Psi_{L}+(1+\mu g(x)) u_{n}^{2} \Psi_{L}+\nabla u_{n} \nabla \Psi_{L} u_{n}\right] d x } \\
& =o_{n}(1)+\int_{\mathbb{R}^{N}} \widetilde{f}\left(u_{n}\right) u_{n} \Psi_{L} d x \\
& \leq o_{n}(1)+\frac{1}{\kappa} \int_{\mathbb{R}^{N}}(1+\mu g(x)) u_{n}^{2} \Psi_{L} d x \leq o_{n}(1)+\frac{1}{2} \int_{\mathbb{R}^{N}}(1+\mu g(x)) u_{n}^{2} \Psi_{L} d x .
\end{aligned}
$$

Then we derive that

$$
\begin{aligned}
\frac{1}{2} \int_{\mathbb{R}^{N}} u_{n}^{2} \Psi_{L} d x & \leq o_{n}(1)-\int_{\mathbb{R}^{N}} \nabla u_{n} \nabla \Psi_{L} u_{n} d x \\
& \leq o_{n}(1)+\frac{C}{L}\left(\int_{\mathbb{R}^{N}}\left|\nabla u_{n}\right|^{2} d x\right)^{1 / 2}\left(\int_{\mathbb{R}^{N}} u_{n}^{2} d x\right)^{1 / 2} .
\end{aligned}
$$

Choosing $L>0$ sufficiently large, we obtain that $\int_{|x| \geq 2 L} u_{n}^{2} d x \leq \beta / 2$. Together with $\int_{B_{1}\left(z_{n}\right)} u_{n}^{2} d x \geq \beta$, we know that the sequence $\left\{z_{n}\right\}$ is bounded in $\mathbb{R}^{N}$.

Now we can prove the existence of critical points of the functional $J_{\mu}$ on $E$.

Lemma 2.3. For $\mu>0$, there is a positive critical point $u_{\mu}$ of $J_{\mu}$ satisfying

$$
\left\|u_{\mu}\right\|_{2^{*}}^{2}<\left[\frac{(N-2) S}{8(N-1)}\right]^{(N-2) / 2} .
$$

Moreover,

$$
J_{\mu}\left(u_{\mu}\right)<\frac{\theta-2}{4 \theta}\left[\frac{N-2}{8(N-1)}\right]^{(N-2) / 2} S^{N / 2} .
$$

Proof. By Lemmas 2.1 and 2.2, there is a sequence $\left\{u_{n}\right\} \subset E$ such that $u_{n} \rightarrow u_{\mu} \neq 0$ weakly in $E$,

$$
J_{\mu}\left(u_{n}\right) \rightarrow c_{\mu}<\frac{\theta-2}{4 \theta}\left[\frac{N-2}{8(N-1)}\right]^{(N-2) / 2} S^{N / 2} \quad \text { and } \quad J_{\mu}^{\prime}\left(u_{n}\right) \rightarrow 0 .
$$


Now we prove that $J_{\mu}^{\prime}\left(u_{\mu}\right)=0$. In fact, we only need to prove that $\left(J_{\mu}^{\prime}\left(u_{\mu}\right), \varphi\right)=$ 0 for all $\varphi \in C_{c}^{\infty}\left(\mathbb{R}^{N}\right)$.

From Theorem A.4 in [37], we know if $1 \leq p, q, r, s<\infty, k \in \mathbb{C}(\bar{\Omega} \times \mathbb{R})$ and $|k(x, u)| \leq C\left(|u|^{p / r}+|u|^{q / s}\right)$, then the operator

$$
A: L^{p}(\Omega) \cap L^{q}(\Omega) \rightarrow L^{r}(\Omega)+L^{s}(\Omega), \quad u \mapsto k(x, u)
$$

is continuous. Here the norm of $L^{p}(\Omega) \cap L^{q}(\Omega)$ is defined by

$$
|u|_{p \wedge q}:=\|u\|_{L^{p}(\Omega)}+\|u\|_{L^{q}(\Omega)}
$$

and the norm of $L^{r}(\Omega)+L^{s}(\Omega)$ is defined by

$$
|u|_{r \vee s}:=\inf \left\{\|v\|_{L^{r}(\Omega)}+\|w\|_{L^{s}(\Omega)} ; v \in L^{r}(\Omega), w \in L^{s}(\Omega), u=v+w\right\} .
$$

We may assume that $u_{n} \geq 0$ in $H$. From (2.3), we know that

$$
\left|k\left(x, u_{n}\right)\right| \leq\left|f\left(u_{n}\right)\right| \leq C\left(\left|u_{n}\right|^{2 / 2}+\left|u_{n}\right|^{t / s}\right)
$$

with $2^{*}-1<t<2^{*}$ and $s=t / 2^{*}-1$. Note that the function $\varphi \in C_{c}^{\infty}\left(\mathbb{R}^{N}\right)$ has a compact support $\Omega_{\varphi}$. Due to $u_{n} \rightarrow u_{\mu}$ weakly in $E$, we have $u_{n} \rightarrow u_{\mu}$ in $L^{2}\left(\Omega_{\varphi}\right) \cap L^{t}\left(\Omega_{\varphi}\right)$. Then by Theorem A.4 in [37], we have $k\left(x, u_{n}\right) \rightarrow k\left(x, u_{\mu}\right)$ in $L^{2}\left(\Omega_{\varphi}\right)+L^{s}\left(\Omega_{\varphi}\right)$. Thus, for all $\varphi \in C_{c}^{\infty}\left(\mathbb{R}^{N}\right)$,

$$
\begin{aligned}
\int_{\mathbb{R}^{N}}\left|k\left(x, u_{n}\right)-k\left(x, u_{\mu}\right)\right||\varphi| d x & =\int_{\Omega_{\varphi}}\left|k\left(x, u_{n}\right)-k\left(x, u_{\mu}\right)\right||\varphi| d x \\
& \leq\left|k\left(x, u_{n}\right)-k\left(x, u_{\mu}\right)\right|_{2 \vee s}|\varphi|_{2 \wedge s^{\prime}},
\end{aligned}
$$

where $1 / s+1 / s^{\prime}=1$. Let $n \rightarrow \infty$, there holds

$$
\lim _{n \rightarrow \infty} \int_{\mathbb{R}^{N}}\left|k\left(x, u_{n}\right)-k\left(x, u_{\mu}\right)\right||\varphi| d x=0 .
$$

Together with

$$
\lim _{n \rightarrow \infty} \int_{\mathbb{R}^{N}}\left[\nabla u_{n} \nabla \varphi+(1+\mu g(x)) u_{n} \varphi\right] d x=\int_{\mathbb{R}^{N}}\left[\nabla u_{\mu} \nabla \varphi+(1+\mu g(x)) u_{\mu} \varphi\right] d x,
$$

we have $J_{\mu}^{\prime}\left(u_{\mu}\right)=0$. A standard argument shows that $u_{\mu}$ is positive.

Since $\left\|u_{n}\right\|_{\mu}$ is bounded, by (2.4), we have

$$
\left\|u_{\mu}\right\|_{\mu}^{2} \leq \frac{4 \theta}{\theta-2} c_{\mu}<\left[\frac{N-2}{8(N-1)}\right]^{(N-2) / 2} S^{N / 2} .
$$

The Sobolev embedding theorem implies that

$$
\left.\left\|u_{\mu}\right\|_{2^{*}}^{2}<\left[\frac{(N-2) S}{8(N-1)}\right)\right]^{(N-2) / 2} .
$$

On the other hand,

$$
c_{\mu}+o_{n}(1)=J_{\mu}\left(u_{n}\right)-\frac{1}{2}\left(J_{\mu}^{\prime}\left(u_{n}\right), u_{n}\right)=\int_{\mathbb{R}^{N}}\left[\frac{1}{2} k\left(x, u_{n}\right) u_{n}-K\left(x, u_{n}\right)\right] d x .
$$


Observe that $k\left(x, u_{n}\right) u_{n} / 2-K\left(x, u_{n}\right) \geq 0$. Then by the Fatou lemma, there holds

$$
J_{\mu}\left(u_{\mu}\right) \leq c_{\mu}<\frac{\theta-2}{4 \theta}\left[\frac{N-2}{8(N-1)}\right]^{(N-2) / 2} S^{N / 2} .
$$

The following lemma is focused on a property of the sequence $\left\{u_{\mu}\right\}$.

Lemma 2.4. For $p_{0} \in\left(2,2^{*}\right)$,

$$
\left\|u_{\mu}\right\|_{\infty} \leq \widetilde{C}\left(N, S, p_{0}\right)\left(1+\left\|u_{\mu}\right\|_{2^{*}}^{\left(2^{*}-2\right) / 2}+\left\|u_{\mu}\right\|_{2^{*}}^{\left(p_{0}-2\right) / 2}\right)^{\delta_{0}^{2} /\left(1-\delta_{0}\right)}\left\|u_{\mu}\right\|_{2^{*}},
$$

where $\widetilde{C}\left(N, S, p_{0}\right)$ is a positive constant and $\delta_{0}:=22^{*} /\left(\left(2^{*}\right)^{2}-22^{*}+4\right)$.

Proof. For simplicity, we denote $u_{\mu}=u$. The basic idea is the Moser iterations. By Lemma 2.3,

$$
\int_{\mathbb{R}^{N}}(\nabla u \nabla \varphi+(1+\mu g(x)) u \varphi) d x=\int_{\mathbb{R}^{N}} k(x, u) \varphi d x, \quad \text { for all } \varphi \in E .
$$

Define $u_{l}=\min \{u, l\}$. Let $z_{l}=u_{l}^{2(\beta-1)} u$ with $\beta>1$. Note that $z_{l} \in E$ if $u \in E$. For $0<r_{2}<r_{1}$ and $y \in \mathbb{R}^{N}$, define $\eta \in C_{0}^{\infty}\left(B_{r_{1}}(y)\right)$ such that $\eta(x)=1$ for $x \in B_{r_{2}}(y), 0 \leq \eta(x) \leq 1$ and $|\nabla \eta| \leq 2 /\left(r_{1}-r_{2}\right)$. Set $\varphi=\eta^{2} z_{l}$ in (2.9). Then

$$
\begin{aligned}
\int_{\mathbb{R}^{N}}\left[\nabla u \nabla\left(u_{l}^{2(\beta-1)} u\right) \eta^{2}+\nabla u \nabla \eta 2 \eta u_{l}^{2(\beta-1)} u+\right. & \left.(1+\mu g(x)) u^{2} u_{l}^{2(\beta-1)} \eta^{2}\right] d x \\
& =\int_{\mathbb{R}^{N}} k(x, u) u u_{l}^{2(\beta-1)} \eta^{2} d x .
\end{aligned}
$$

Conditions $\left(\mathrm{f}_{1}\right)-\left(\mathrm{f}_{3}\right)$ imply that for all $\varepsilon>0$, there exists $C(\varepsilon)>0$ such that $k(x, u) u \leq f(u) u \leq \varepsilon|u|^{2}+(1+\varepsilon)|u|^{2^{*}}+C(\varepsilon)|u|^{p_{0}}$. Choose $\varepsilon_{0} \in(0,1 / 2)$. Then

$$
\begin{gathered}
\int_{\mathbb{R}^{N}}\left[|\nabla u|^{2} u_{l}^{2(\beta-1)} \eta^{2}+2(\beta-1)\left|\nabla u_{l}\right|^{2} u_{l}^{2(\beta-1)} \eta^{2}+\nabla u \nabla \eta 2 \eta u_{l}^{2(\beta-1)} u\right] d x \\
\leq\left(1+\varepsilon_{0}\right) \int_{\mathbb{R}^{N}}|u|^{2^{*}} u_{l}^{2(\beta-1)} \eta^{2} d x+C\left(\varepsilon_{0}\right) \int_{\mathbb{R}^{N}}|u|^{p_{0}} u_{l}^{2(\beta-1)} \eta^{2} d x .
\end{gathered}
$$

By the Young inequality,

$$
\left|\int_{\mathbb{R}^{N}} \nabla u \nabla \eta \eta u_{l}^{2(\beta-1)} u d x\right| \leq \frac{1}{4} \int_{\mathbb{R}^{N}}|\nabla u|^{2} u_{l}^{2(\beta-1)} \eta^{2} d x+4 \int_{\mathbb{R}^{N}}|\nabla \eta|^{2}|u|^{2} u_{l}^{2(\beta-1)} d x .
$$

Together with (2.10), there holds

$$
\begin{aligned}
& \int_{\mathbb{R}^{N}}\left[|\nabla u|^{2} u_{l}^{2(\beta-1)} \eta^{2}+2(\beta-1)\left|\nabla u_{l}\right|^{2} u_{l}^{2(\beta-1)} \eta^{2}\right] d x \\
& \leq 16 \int_{\mathbb{R}^{N}}|\nabla \eta|^{2}|u|^{2} u_{l}^{2(\beta-1)} d x+2\left(1+\varepsilon_{0}\right) \int_{\mathbb{R}^{N}}|u|^{2^{*}} u_{l}^{2(\beta-1)} \eta^{2} d x \\
&+2 C\left(\varepsilon_{0}\right) \int_{\mathbb{R}^{N}}|u|^{p_{0}} u_{l}^{2(\beta-1)} \eta^{2} d x .
\end{aligned}
$$


By the Sobolev embedding theorem,

$$
\begin{aligned}
& S\left\|\eta u u_{l}^{\beta-1}\right\|_{2^{*}}^{2} \leq \int_{\mathbb{R}^{N}}\left|\nabla\left(\eta u u_{l}^{\beta-1}\right)\right|^{2} d x \\
& \leq 2 \int_{\mathbb{R}^{N}}|\nabla \eta|^{2}|u|^{2} u_{l}^{2(\beta-1)} d x+2 \int_{\mathbb{R}^{N}} \eta^{2} t\left|\nabla\left(u u_{l}^{\beta-1}\right)\right|^{2} d x \\
& \leq(\beta+1) \int_{\mathbb{R}^{N}}\left[|\nabla u|^{2} u_{l}^{2(\beta-1)} \eta^{2}+2(\beta-1)\left|\nabla u_{l}\right|^{2} u_{l}^{2(\beta-1)} \eta^{2}\right] d x \\
& \quad+2 \int_{\mathbb{R}^{N}}|\nabla \eta|^{2}|u|^{2} u_{l}^{2(\beta-1)} d x \\
& \leq 17(\beta+1) \int_{\mathbb{R}^{N}}|\nabla \eta|^{2}|u|^{2} u_{l}^{2(\beta-1)} d x \\
& \quad+2(\beta+1) C\left(\varepsilon_{0}\right) \int_{\mathbb{R}^{N}}|u|^{p_{0}} u_{l}^{2(\beta-1)} \eta^{2} d x \\
& \quad+2(\beta+1)\left(1+\varepsilon_{0}\right) \int_{\mathbb{R}^{N}}|u|^{2^{*}} u_{l}^{2(\beta-1)} \eta^{2} d x .
\end{aligned}
$$

Lemma 2.3 implies that $\left(2^{*}+2\right)\|u\|_{2^{*}}^{2^{*}-2}<S / 2$. Since $\varepsilon_{0} \in(0,1 / 2)$, we have $\left(2^{*}+2\right)\left(1+\varepsilon_{0}\right)\|u\|_{2^{*}}^{2^{*}-2}<3 S / 4$. We also have

$$
\int_{\mathbb{R}^{N}}|u|^{2^{*}} u_{l}^{2(\beta-1)} \eta^{2} d x \leq\|u\|_{2^{*}}^{2^{*}-2}\left\|u u_{l}^{\beta-1} \eta\right\|_{2^{*}}^{2}
$$

Set $\beta=\beta_{0}=2^{*} / 2$ in $(2.11)$. Then

$$
\begin{aligned}
S\left\|\eta u u_{l}^{\beta_{0}-1}\right\|_{2^{*}}^{2} \leq & 68\left(\beta_{0}+1\right) \int_{\mathbb{R}^{N}}|\nabla \eta|^{2}|u|^{2} u_{l}^{2\left(\beta_{0}-1\right)} d x \\
& +8\left(\beta_{0}+1\right) C\left(\varepsilon_{0}\right) \int_{\mathbb{R}^{N}}|u|^{p_{0}} u_{l}^{2\left(\beta_{0}-1\right)} \eta^{2} d x \\
\leq & 136 \beta_{0} \int_{\mathbb{R}^{N}}|\nabla \eta|^{2}|u|^{2} u_{l}^{2\left(\beta_{0}-1\right)} d x \\
& +16 \beta_{0} C\left(\varepsilon_{0}\right)\left\|\eta u u_{l}^{\beta_{0}-1}\right\|_{2^{*}}^{2}\|u\|_{2^{*}}^{p_{0}-2}\left|B_{r_{1}}(y)\right|^{\left(2^{*}-p_{0}\right) / 2^{*}}
\end{aligned}
$$

Note that we can find $r_{1} \in(0,1)$ such that

$$
\begin{aligned}
& \left|B_{r_{1}}(y)\right| \\
\leq & \min \left\{1, S^{2^{*} /\left(2^{*}-p_{0}\right)}\left(32 \beta_{0} C\left(\varepsilon_{0}\right)\right)^{-2^{*} /\left(2^{*}-p_{0}\right)}\left[\frac{S}{2\left(2^{*}+2\right)}\right]^{-N\left(p_{0}-2\right) /\left(2\left(2^{*}-p_{0}\right)\right)}\right\} .
\end{aligned}
$$

Recall that $\left(2^{*}+2\right)\|u\|_{2^{*}}^{2^{*}-2}<S / 2$, so we have

$$
\left|B_{r_{1}}(y)\right| \leq S^{2^{*} /\left(2^{*}-p_{0}\right)}\left(32 \beta_{0} C\left(\varepsilon_{0}\right)\right)^{-2^{*} /\left(2^{*}-p_{0}\right)}\|u\|_{2^{*}}^{-2^{*}\left(p_{0}-2\right) /\left(2^{*}-p_{0}\right)} .
$$


Together with (2.12), there holds

$$
\begin{aligned}
& \left\|u u_{l}^{\beta_{0}-1}\right\|_{L^{2^{*}\left(B_{r_{2}}(y)\right)}}^{2} \leq\left\|u u_{l}^{\beta_{0}-1} \eta\right\|_{2^{*}}^{2} \\
& \leq \frac{272}{S} \beta_{0} \int_{\mathbb{R}^{N}}|\nabla \eta|^{2}|u|^{2} u_{l}^{2\left(\beta_{0}-1\right)} d x \leq \frac{272}{S} \beta_{0}\left(\frac{2}{r_{1}-r_{2}}\right)^{2}\left\|u u_{l}^{\beta_{0}-1}\right\|_{L^{2}\left(B_{r_{1}}(y)\right)}^{2} .
\end{aligned}
$$

Let $l \rightarrow \infty$. We have

$$
\|u\|_{L^{\beta_{0} 2^{*}}\left(B_{r_{2}}(y)\right)} \leq\left(\frac{C_{0}}{r_{1}-r_{2}}\right)^{1 / \beta_{0}}\|u\|_{L^{2 *}\left(B_{r_{1}}(y)\right)},
$$

where $C_{0}=2 \sqrt{272 \beta_{0} / S}$. For $i \geq 2$, let $r_{i}=\left(2+2^{-i}\right) r_{1} / 4$. Define $\eta_{i} \in$ $C_{0}^{\infty}\left(B_{r_{i}}(y)\right)$ such that $\eta_{i}(x)=1$ for $x \in B_{r_{i+1}}(y), 0 \leq \eta_{i}(x) \leq 1$ and $\left|\nabla \eta_{i}\right| \leq$ $2 /\left(r_{i}-r_{i+1}\right)$. Let $\beta_{i}=\delta_{0}^{-i}$, where $\delta_{0}=22^{*} /\left(\left(2^{*}\right)^{2}-22^{*}+4\right)$. Then $\beta_{i}>1$. Similarly to $(2.11)$, we have

$$
\begin{aligned}
& S\left\|\eta_{i} u u_{l}^{\beta_{i}-1}\right\|_{2^{*}}^{2} \leq C_{1}\left(\beta_{i}+1\right) \int_{\mathbb{R}^{N}}\left|\nabla \eta_{i}\right|^{2}|u|^{2} u_{l}^{2\left(\beta_{i}-1\right)} d x \\
& \quad+C_{1}\left(\beta_{i}+1\right) \int_{\mathbb{R}^{N}}|u|^{p_{0}} u_{l}^{2\left(\beta_{i}-1\right)} \eta_{i}^{2} d x+C_{1}\left(\beta_{i}+1\right) \int_{\mathbb{R}^{N}}|u|^{2^{*}} u_{l}^{2\left(\beta_{i}-1\right)} \eta_{i}^{2} d x,
\end{aligned}
$$

where $C_{1}=\max \left\{17,2 C\left(\varepsilon_{0}\right)\right\}$. Then, due to $\left|B_{r_{i}}(y)\right|<1$ and $(2.13)$,

$$
\begin{aligned}
& S\left\|u u_{l}^{\beta_{i}-1}\right\|_{L^{2^{*}\left(B_{r_{i+1}}(y)\right)}}^{2} \leq C_{1}\left(\beta_{i}+1\right)\left(\frac{2}{r_{i}-r_{i+1}}\right)^{2}\left\|u u_{l}^{\beta_{i}-1}\right\|_{L^{2^{*} \delta_{0}\left(B_{r_{i}}(y)\right)}}^{2} \\
& +C_{1}\left(\beta_{i}+1\right)\left[\|u\|_{L^{2 * \beta_{0}\left(B_{r_{i}}(y)\right)}}^{2^{*}-2}+\|u\|_{L^{2 * \beta_{0}\left(B_{r_{i}}(y)\right)}}^{p_{0}-2}\right]\left\|u u_{l}^{\beta_{i}-1}\right\|_{L^{2 * \delta_{0}\left(B_{r_{i}}(y)\right)}}^{2} \\
& \leq 2 C_{1} \beta_{i}^{2}\left(\frac{2}{r_{i}-r_{i+1}}\right)^{2}\left\|u u_{l}^{\beta_{i}-1}\right\|_{L^{2^{*} \delta_{0}\left(B_{r_{i}}(y)\right)}}^{2} \\
& +2 C_{1} \beta_{i}^{2}\left\|u u_{l}^{\beta_{i}-1}\right\|_{L^{2^{*} \delta_{0}\left(B_{r_{i}}(y)\right)}}^{2}\left(\frac{C_{0}}{r_{1}-r_{2}}\right)^{\left(2^{*}-2\right) / \beta_{0}}\|u\|_{L^{2^{*}}\left(B_{r_{1}}(y)\right)}^{2^{*}-2} \\
& +2 C_{1} \beta_{i}^{2}\left\|u u_{l}^{\beta_{i}-1}\right\|_{L^{2^{*} \delta_{0}\left(B_{r_{i}}(y)\right)}}^{2}\left(\frac{C_{0}}{r_{1}-r_{2}}\right)^{\left(p_{0}-2\right) / \beta_{0}}\|u\|_{L^{2^{*}\left(B_{r_{1}}(y)\right)}}^{p_{0}-2} .
\end{aligned}
$$

Due to $1 /\left(r_{i}-r_{i+1}\right)=4 \cdot 2^{i+1} / r_{1}$ and $\left(2^{*}-2\right) / \beta_{0},\left(p_{0}-2\right) / \beta_{0}<2$, we can find $C_{2}>0$ such that $\left(C_{0} /\left(r_{1}-r_{2}\right)\right)^{\left(2^{*}-2\right) / \beta_{0}},\left(C_{0} /\left(r_{1}-r_{2}\right)\right)^{\left(p_{0}-2\right) / \beta_{0}} \leq$ $\left(C_{2} /\left(r_{i}-r_{i+1}\right)\right)^{2}$. Thus,

$$
\begin{aligned}
& S\left\|u u_{l}^{\beta_{i}-1}\right\|_{L^{2^{*}\left(B_{r_{i+1}}(y)\right)}}^{2} \leq \frac{8 C_{1} \beta_{i}^{2}}{\left(r_{i}-r_{i+1}\right)^{2}}\left\|u u_{l}^{\beta_{i}-1}\right\|_{L^{2^{*} \delta_{0}\left(B_{r_{i}}(y)\right)}}^{2} \\
& +\frac{2 C_{1} C_{2}^{2} \beta_{i}^{2}}{\left(r_{i}-r_{i+1}\right)^{2}}\left(\|u\|_{L^{2^{*}}\left(B_{r_{1}}(y)\right)}^{2^{*}-2}+\|u\|_{L^{2^{*}}\left(B_{r_{1}}(y)\right)}^{p_{0}-2}\right)\left\|u u_{l}^{\beta_{i}-1}\right\|_{L^{2^{*} \delta_{0}\left(B_{r_{i}}(y)\right)}}^{2} \\
& \leq\left[\frac{C_{3} \beta_{i}\left(1+\|u\|_{L^{2^{*}\left(B_{r_{1}}(y)\right)}}^{\left(2^{*}-2\right) / 2}+\|u\|_{L^{2^{*}}\left(B_{r_{1}}(y)\right)}^{\left(p_{0}-2\right) / 2}\right)}{r_{i}-r_{i+1}}\right]^{2}\left\|u u_{l}^{\beta_{i}-1}\right\|_{L^{2^{*} \delta_{0}\left(B_{r_{i}}(y)\right)}}^{2},
\end{aligned}
$$


where $C_{3}=C_{3}\left(N, S, p_{0}\right)$ in view of the definition of $C_{0}, C_{1}$ and $C_{2}$. Let $l \rightarrow \infty$, we have

(2.14) $\|u\|_{L^{2^{*} \beta_{i}\left(B_{r_{i+1}}(y)\right)}}$

$$
\leq\left[\frac{\bar{C} \beta_{i}\left(1+\|u\|_{2^{*}}^{\left(2^{*}-2\right) / 2}+\|u\|_{2^{*}}^{\left(p_{0}-2\right) / 2}\right)}{r_{i}-r_{i+1}}\right]^{1 / \beta_{i}}\|u\|_{L^{2^{*} \beta_{i-1}\left(B_{r_{i}}(y)\right)}},
$$

where $\bar{C}=C_{3} / \sqrt{S}$. Then, by $(2.14)$,

$$
\begin{aligned}
& \|u\|_{L^{2^{*} \beta_{i}}\left(B_{r_{i+1}}(y)\right)} \\
\leq & \prod_{j=2}^{i}\left[\frac{\bar{C} \beta_{j}\left(1+\|u\|_{2^{*}}^{\left(2^{*}-2\right) / 2}+\|u\|_{2^{*}}^{\left(p_{0}-2\right) / 2}\right)}{r_{j}-r_{j+1}}\right]^{1 / \beta_{j}}\|u\|_{L^{2^{*} \beta_{1}}\left(B_{r_{2}}(y)\right)} \\
= & \prod_{j=2}^{i}\left[\frac{8 \bar{C}\left(1+\|u\|_{2^{*}}^{\left(2^{*}-2\right) / 2}+\|u\|_{2^{*}}^{\left(p_{0}-2\right) / 2}\right)}{r_{1}}\left(\frac{2}{\delta_{0}}\right)^{j}\right]^{\delta_{0}^{j}}\|u\|_{L^{2^{*} \beta_{1}}\left(B_{r_{2}}(y)\right)} \\
= & \left(\frac{2}{\delta_{0}}\right)^{\left[2 \delta_{0}^{2} /\left(1-\delta_{0}\right)+\delta_{0}^{3}\left(1-\delta_{0}^{i-2}\right) /\left(1-\delta_{0}\right)^{2}-i \delta_{0}^{i+1} /\left(1-\delta_{0}\right)\right]}\|u\|_{L^{2^{*} \beta_{0}-2^{*}+2}\left(B_{r_{2}}(y)\right)} \\
& \times\left[\frac{8 \bar{C}\left(1+\|u\|_{2^{*}}^{\left(2^{*}-2\right) / 2}+\|u\|_{2^{*}}^{\left(p_{0}-2\right) / 2}\right)}{r_{1}}\right]^{\delta_{0}^{2}\left(1-\delta_{0}^{i-1}\right) /\left(1-\delta_{0}\right)} .
\end{aligned}
$$

Let $i \rightarrow \infty$. We have

$$
\begin{aligned}
\|u\|_{L^{\infty}\left(B_{r_{1} / 2}(y)\right)} \leq\left(\frac{2}{\delta_{0}}\right)^{\delta_{0}^{2}\left(2-\delta_{0}\right) /\left(1-\delta_{0}\right)^{2}} & \\
& \times\left[\frac{8 \bar{C}\left(1+\|u\|_{2^{*}}^{\left(2^{*}-2\right) / 2}+\|u\|_{2^{*}}^{\left(p_{0}-2\right) / 2}\right)}{r_{1}}\right]^{\delta_{0}^{2} /\left(1-\delta_{0}\right)}\|u\|_{L^{2^{*} \beta_{0}\left(B_{r_{2}}(y)\right)}} \\
\leq & \left(\frac{2}{\delta_{0}}\right)^{\delta_{0}^{2}\left(2-\delta_{0}\right) /\left(1-\delta_{0}\right)^{2}}\left(\frac{16 C_{0}}{7 r_{1}}\right)^{1 / \beta_{0}} \\
& \times\left[\frac{8 \bar{C}\left(1+\|u\|_{2^{*}}^{\left(2^{*}-2\right) / 2}+\|u\|_{2^{*}}^{\left(p_{0}-2\right) / 2}\right)}{r_{1}}\right]^{\delta_{0}^{2} /\left(1-\delta_{0}\right)}\|u\|_{2^{*}} \\
= & C\left(N, S, p_{0}\right)\left(1+\|u\|_{2^{*}}^{\left(2^{*}-2\right) / 2}+\|u\|_{2^{*}}^{\left(p_{0}-2\right) / 2}\right)^{\delta_{0}^{2} /\left(1-\delta_{0}\right)}\|u\|_{2^{*}},
\end{aligned}
$$

in view of $2^{*} \beta_{0}-2^{*}+2<2^{*} \beta_{0},\left|B_{r_{2}}(y)\right| \leq 1$ and (2.13). Now Lemma 2.4 follows easily.

\section{Proofs of Theorems 1.1-1.3}

Now we are ready to give the proof of Theorem 1.1.

Proof of Theorem 1.1. By Lemma 2.3, there exists $u_{\mu} \in E, u_{\mu}>0$, such that

$$
J_{\mu}^{\prime}\left(u_{\mu}\right)=0 \quad \text { and } \quad J_{\mu}\left(u_{\mu}\right)<\frac{\theta-2}{4 \theta}\left[\frac{N-2}{8(N-1)}\right]^{(N-2) / 2} S^{N / 2} .
$$


Moreover,

Then, by Lemma 2.4,

$$
\left\|u_{\mu}\right\|_{2^{*}}<\left[\frac{(N-2) S}{8(N-1)}\right]^{(N-2) / 4} .
$$

$$
\begin{array}{r}
\left\|u_{\mu}\right\|_{\infty} \leq \widetilde{C}\left(N, S, p_{0}\right)\left(1+\left\|u_{\mu}\right\|_{2^{*}}^{\left(2^{*}-2\right) / 2}+\left\|u_{\mu}\right\|_{2^{*}}^{\left(p_{0}-2\right) / 2}\right)^{\delta_{0}^{2} /\left(1-\delta_{0}\right)}\left\|u_{\mu}\right\|_{2^{*}} \\
\leq \widetilde{C}_{0}\left(N, S, p_{0}\right):=\widetilde{C}_{0} .
\end{array}
$$

From (2.3) and (3.1), we can find $\mu_{0}>0$ such that for $\mu>\mu_{0}, f\left(u_{\mu}\right) / u_{\mu} \leq$ $(1+\mu g(x)) / \kappa$ for all $|x| \geq R$, from which we have $k\left(x, u_{\mu}\right)=f\left(u_{\mu}\right)$. Then

$$
I_{\mu}^{\prime}\left(u_{\mu}\right)=0 \quad \text { and } \quad I_{\mu}\left(u_{\mu}\right)=J_{\mu}\left(u_{\mu}\right)<\frac{\theta-2}{4 \theta}\left[\frac{N-2}{8(N-1)}\right]^{(N-2) / 2} S^{N / 2} .
$$

Let $m_{\mu}:=\inf \left\{I_{\mu}(v): v \in E, v>0, I_{\mu}^{\prime}(v)=0\right\}$. Since $I_{\mu}^{\prime}\left(u_{\mu}\right)=0$, we have

$$
m_{\mu} \leq I_{\mu}\left(u_{\mu}\right)<\frac{\theta-2}{4 \theta}\left[\frac{N-2}{8(N-1)}\right]^{(N-2) / 2} S^{N / 2} .
$$

By the definition of $m_{\mu}$, there exists $\left\{v_{n}\right\} \subset E$ such that $v_{n}>0, I_{\mu}\left(v_{n}\right) \rightarrow m_{\mu}$ and $I_{\mu}^{\prime}\left(v_{n}\right)=0$. Note that for $n$ large enough,

$$
I_{\mu}\left(v_{n}\right)<\frac{\theta-2}{4 \theta}\left[\frac{N-2}{8(N-1)}\right]^{(N-2) / 2} S^{N / 2} .
$$

Without loss of generality, we assume that

$$
I_{\mu}\left(v_{n}\right)<\frac{\theta-2}{4 \theta}\left[\frac{N-2}{8(N-1)}\right]^{(N-2) / 2} S^{N / 2}
$$

for all $n$. Then by $\left(\mathrm{f}_{4}\right)$,

$$
\frac{\theta-2}{4 \theta}\left[\frac{N-2}{8(N-1)}\right]^{(N-2) / 2} S^{N / 2}>I_{\mu}\left(v_{n}\right)-\frac{1}{\theta}\left(I_{\mu}^{\prime}\left(v_{n}\right), v_{n}\right) \geq\left(\frac{1}{2}-\frac{1}{\theta}\right)\left\|v_{n}\right\|_{\mu}^{2},
$$

which implies that

$$
\left\|v_{n}\right\|_{\mu}^{2} \leq \frac{1}{2}\left[\frac{N-2}{8(N-1)}\right]^{(N-2) / 2} S^{N / 2} .
$$

Applying the Sobolev embedding theorem, we have

$$
\left\|v_{n}\right\|_{2^{*}}^{2}<\left[\frac{(N-2) S}{8(N-1)}\right]^{(N-2) / 2} .
$$

Due to $I_{\mu}^{\prime}\left(v_{n}\right)=0$, there holds

$$
\int_{\mathbb{R}^{N}}\left(\nabla v_{n} \nabla \varphi+(1+\mu g(x)) v_{n} \varphi\right) d x=\int_{\mathbb{R}^{N}} f\left(v_{n}\right) \varphi d x, \quad \text { for all } \varphi \in E .
$$

Following the same lines as in the proof of Lemma 2.4, we get

$$
\left\|v_{n}\right\|_{\infty} \leq \widetilde{C}\left(N, S, p_{0}\right)\left(1+\left\|v_{n}\right\|_{2^{*}}^{\left(2^{*}-2\right) / 2}+\left\|v_{n}\right\|_{2^{*}}^{\left(p_{0}-2\right) / 2}\right)^{\delta_{0}^{2} /\left(1-\delta_{0}\right)}\left\|v_{n}\right\|_{2^{*}} \leq \widetilde{C}_{0},
$$


where $\widetilde{C}\left(N, S, p_{0}\right)$ is as in Lemma 2.4. Then for $\mu>\mu_{0}$, we obtain $f\left(v_{n}\right) / v_{n} \leq$ $(1+\mu g(x)) / \kappa$ for all $|x| \geq R$, from which we have $f\left(v_{n}\right)=k\left(x, v_{n}\right)$. Thus, $I_{\mu}\left(v_{n}\right)=J_{\mu}\left(v_{n}\right)$ and $I_{\mu}^{\prime}\left(v_{n}\right)=J_{\mu}^{\prime}\left(v_{n}\right)$. From $(2.3)$ and $\left(I_{\mu}^{\prime}\left(v_{n}\right), v_{n}\right)=0$, we have

$$
\left\|v_{n}\right\|_{\mu}^{2} \leq \varepsilon \int_{\mathbb{R}^{N}}\left|v_{n}\right|^{2} d x+C(\varepsilon) \int_{\mathbb{R}^{N}}\left|v_{n}\right|^{2^{*}} d x
$$

Set $\varepsilon=1 / 2$. The Sobolev embedding theorem implies that

$$
\frac{1}{2}\left\|v_{n}\right\|_{\mu}^{2} \leq C\left(\frac{1}{2}\right) \int_{\mathbb{R}^{N}}\left|v_{n}\right|^{2^{*}} d x \leq C\left\|v_{n}\right\|_{\mu}^{2^{*}}
$$

from which we have

$$
\left\|v_{n}\right\|_{\mu} \geq \frac{1}{(2 C)^{1 /\left(2^{*}-2\right)}}:=\rho>0 .
$$

Now we claim that there is a bounded sequence $\left\{\bar{z}_{n}\right\} \subset \mathbb{R}^{N}$ and $\bar{\beta}>0$ such that $\int_{B_{1}\left(\bar{z}_{n}\right)} v_{n}^{2} d x \geq \bar{\beta}$. In fact, if $\lim _{n \rightarrow \infty} \sup _{\bar{z} \in \mathbb{R}^{N}} \int_{B_{1}(\bar{z})} v_{n}^{2} d x=0$, then similar to (2.7)-(2.8), we can prove that

$$
o_{n}(1) \geq\left\|v_{n}\right\|_{\mu}^{2}-\int_{\mathbb{R}^{N}}\left\|v_{n}\right\|^{2^{*}} d x
$$

and

$$
m_{\mu}+o_{n}(1) \geq \frac{1}{N} \int_{\mathbb{R}^{N}}\left|\nabla u_{n}\right|^{2} d x+\frac{1}{2 N} \int_{\mathbb{R}^{N}}(1+\mu g(x)) u_{n}^{2} d x .
$$

By (3.3), we may assume that

$\lim _{n \rightarrow \infty}\left[\int_{\mathbb{R}^{N}}\left|\nabla v_{n}\right|^{2} d x+\frac{1}{2} \int_{\mathbb{R}^{N}}(1+\mu g(x)) v_{n}^{2} d x\right]=\bar{l} \quad$ and $\quad \lim _{n \rightarrow \infty} \int_{\mathbb{R}^{N}}\left|v_{n}\right|^{2^{*}} d x \geq \bar{l}$.

From (3.2), we know $\bar{l}>0$. The Sobolev embedding theorem implies that

$$
S \leq \frac{\left\|v_{n}\right\|_{\mu}^{2}}{\left(\int_{\mathbb{R}^{N}}\left|v_{n}\right|^{2^{*}} d x\right)^{2 / 2^{*}}},
$$

from which we get $\bar{l} \geq S^{N / 2}$. Together with (3.4), there holds $m_{\mu} \geq S^{N / 2} / N$, a contradiction with

$$
m_{\mu}<\frac{\theta-2}{4 \theta}\left[\frac{N-2}{8(N-1)}\right]^{(N-2) / 2} S^{N / 2}<\frac{2}{8^{N / 2}} S^{N / 2}<\frac{1}{N} S^{N / 2}
$$

for $N \geq 3$. Therefore, there is a sequence $\left\{\bar{z}_{n}\right\} \subset \mathbb{R}^{N}$ and $\bar{\beta}>0$ such that $\int_{B_{1}\left(\bar{z}_{n}\right)} v_{n}^{2} d x \geq \bar{\beta}$. The prove of the sequence $\left\{\bar{z}_{n}\right\}$ is bounded is just the same as in the proof of Lemma 2.2, we omit it here. Thus, we have $v_{n} \rightarrow v_{\mu} \neq 0$ weakly in $E$,

$$
I_{\mu}\left(v_{n}\right)=J_{\mu}\left(v_{n}\right) \rightarrow m_{\mu}<\frac{\theta-2}{4 \theta}\left[\frac{N-2}{8(N-1)}\right]^{(N-2) / 2} S^{N / 2}
$$


and $I_{\mu}^{\prime}\left(v_{n}\right)=J_{\mu}^{\prime}\left(v_{n}\right)=0$. Following the same lines as in the proof of Lemma 2.3, we can derive that $v_{n} \rightarrow v_{\mu}>0$ weakly in $E, I_{\mu}^{\prime}\left(v_{\mu}\right)=0$ and $m_{\mu} \geq I_{\mu}\left(v_{\mu}\right)$. Since $I_{\mu}^{\prime}\left(v_{\mu}\right)=0$, by the definition of $m_{\mu}$, we have $I_{\mu}\left(v_{\mu}\right) \geq m_{\mu}$. Then $I_{\mu}\left(v_{\mu}\right)=m_{\mu}$. Together with $v_{\mu}>0$ and $I_{\mu}^{\prime}\left(v_{\mu}\right)=0$, we know Theorem 1.1 holds.

In Theorem 1.3, we study the behavior of $u_{\mu}$ as $\mu \rightarrow \infty$.

Proof of Theorem 1.3. From the proof of Theorem 1.1, we know that $u_{\mu_{n}}$ satisfies $I_{\mu_{n}}^{\prime}\left(u_{\mu_{n}}\right)=0$,

$$
\begin{aligned}
I\left(u_{\mu_{n}}\right) & <\frac{\theta-2}{4 \theta}\left[\frac{N-2}{8(N-1)}\right]^{(N-2) / 2} S^{N / 2}, \\
\left\|u_{\mu_{n}}\right\|_{\mu_{n}}^{2} & \leq \frac{1}{2}\left[\frac{N-2}{8(N-1)}\right]^{(N-2) / 2} S^{N / 2}
\end{aligned}
$$

and $\left\|u_{\mu_{n}}\right\|_{\infty} \leq \widetilde{C}_{0}$, where $\widetilde{C}_{0}$ is as in Theorem 1.1. For simplicity, denote $u_{\mu_{n}}=u_{\mu}$. Hence $u_{\mu} \rightarrow u$ weakly in $H^{1}\left(\mathbb{R}^{N}\right)$ as $\mu \rightarrow+\infty$. We also have

$$
\mu \int_{\mathbb{R}^{N}} g(x) u_{\mu}^{2} d x \leq\left\|u_{\mu}\right\|_{\mu}^{2} \leq \frac{1}{2}\left[\frac{N-2}{8(N-1)}\right]^{(N-2) / 2} S^{N / 2} .
$$

By the Fatou lemma, we get $\int_{\mathbb{R}^{N}} g(x) u^{2} d x=0$. Then it follows from $\left(\mathrm{G}_{1}\right)-\left(\mathrm{G}_{2}\right)$ that $u(x)=0$ for almost every $x \in \mathbb{R}^{N} \backslash \Omega_{0}$. We claim that

$$
\lim _{\mu \rightarrow+\infty} \int_{\mathbb{R}^{N}} f\left(u_{\mu}\right) u_{\mu} d x=\int_{\mathbb{R}^{N}} f(u) u d x .
$$

Note that $g(x) \geq g_{0}$ for $|x| \geq R$. For $L>R$, define $\Phi_{L} \in C_{0}^{\infty}\left(\mathbb{R}^{N}\right)$ such that $\Phi_{L}(x)=0$ for $|x| \leq L, \Phi_{L}(x)=1$ for $|x| \geq 2 L$ and $0 \leq \Phi_{L}(x) \leq 1$. Moreover, $\left|\nabla \Phi_{L}\right| \leq C / L$. Due to $I_{\mu}^{\prime}\left(u_{\mu}\right)=0$, we have

$$
\int_{\mathbb{R}^{N}}\left[\nabla u_{\mu} \nabla\left(\Phi_{L} u_{\mu}\right)+(1+\mu g(x)) \Phi_{L} u_{\mu}^{2}\right] d x=\int_{\mathbb{R}^{N}} \Phi_{L} f\left(u_{\mu}\right) u_{\mu} d x .
$$

Observe that

$$
\begin{aligned}
\int_{\mathbb{R}^{N}}\left[(1+\mu g(x)) \Phi_{L} u_{\mu}^{2}-\Phi_{L} f\left(u_{\mu}\right) u_{\mu}\right] d x & \\
& \geq \int_{\mathbb{R}^{N}}\left[\left(1+\mu g_{0}\right) \Phi_{L} u_{\mu}^{2}-\Phi_{L} f\left(u_{\mu}\right) u_{\mu}\right] d x .
\end{aligned}
$$

Then, due to $\left\|u_{\mu}\right\|_{\infty} \leq \widetilde{C}_{0}$ and (2.3), we can find $\bar{\mu}>0$ such that for $\mu>\bar{\mu}$,

$$
\int_{\mathbb{R}^{N}}\left[(1+\mu g(x)) \Phi_{L} u_{\mu}^{2}-\Phi_{L} f\left(u_{\mu}\right) u_{\mu}\right] d x \geq \int_{\mathbb{R}^{N}} \Phi_{L} u_{\mu}^{2} d x .
$$

From (3.6)-(3.7),

$$
\int_{|x| \geq 2 L}\left(\left|\nabla u_{\mu}\right|^{2}+u_{\mu}^{2}\right) d x \leq \int_{\mathbb{R}^{N}}\left|\nabla \Phi_{L}\right|\left|\nabla u_{\mu}\right|\left|u_{\mu}\right| d x \leq \frac{C}{L} .
$$


On the other hand, in view of $\left\|u_{\mu}\right\|_{\infty} \leq \widetilde{C}_{0}$, by the Lebesgue Dominated Convergence Theorem, we have

$$
\lim _{\mu \rightarrow+\infty} \int_{|x| \leq 2 L}\left|f\left(u_{\mu}\right) u_{\mu}-f(u) u\right| d x=0 .
$$

Then

$$
\begin{aligned}
\lim _{\mu \rightarrow+\infty} \int_{\mathbb{R}^{N}} \mid f\left(u_{\mu}\right) u_{\mu} & -f(u) u\left|d x=\lim _{\mu \rightarrow+\infty} \int_{|x| \geq 2 L}\right| f\left(u_{\mu}\right) u_{\mu}-f(u) u \mid d x \\
& \leq \int_{|x| \geq 2 L}|f(u) u| d x+\lim _{\mu \rightarrow+\infty} \int_{|x| \geq 2 L}\left|f\left(u_{\mu}\right) u_{\mu}\right| d x
\end{aligned}
$$

By (2.3) and (3.8), we get $\int_{|x| \geq 2 L}\left|f\left(u_{\mu}\right) u_{\mu}\right| d x \leq C / L$. Together with (3.9), we can derive that (3.5) holds. Now we claim that $u_{\mu} \rightarrow u$ in $H^{1}\left(\mathbb{R}^{N}\right)$ as $\mu \rightarrow+\infty$. In fact, due to $I_{\mu}^{\prime}\left(u_{\mu}\right)=0$, we have

$$
\int_{\mathbb{R}^{N}}\left(\nabla u_{\mu} \nabla u+u_{\mu} u\right) d x=\int_{\mathbb{R}^{N}} f\left(u_{\mu}\right) u d x .
$$

Let $\mu \rightarrow+\infty$, we get

$$
\int_{\mathbb{R}^{N}}\left(|\nabla u|^{2}+u^{2}\right) d x=\int_{\mathbb{R}^{N}} f(u) u d x
$$

Due to $I_{\mu}^{\prime}\left(u_{\mu}\right)=0$, we also have

$$
\int_{\mathbb{R}^{N}}\left(\left|\nabla u_{\mu}\right|^{2}+u_{\mu}^{2}\right) d x \leq \int_{\mathbb{R}^{N}}\left[\left|\nabla u_{\mu}\right|^{2}+(1+\mu g(x)) u_{\mu}^{2}\right] d x=\int_{\mathbb{R}^{N}} f\left(u_{\mu}\right) u_{\mu} d x .
$$

Then, by the Fatou lemma, (3.5) and (3.11), we get

$$
\begin{aligned}
\int_{\mathbb{R}^{N}}\left(|\nabla u|^{2}+u^{2}\right) d x & \leq \lim _{\mu \rightarrow+\infty} \int_{\mathbb{R}^{N}}\left(\left|\nabla u_{\mu}\right|^{2}+u_{\mu}^{2}\right) d x \\
& \leq \int_{\mathbb{R}^{N}} f(u) u d x=\int_{\mathbb{R}^{N}}\left(|\nabla u|^{2}+u^{2}\right) d x .
\end{aligned}
$$

The Brezis-Lieb lemma in [37] implies that $u_{\mu} \rightarrow u$ in $H^{1}\left(\mathbb{R}^{N}\right)$ as $\mu \rightarrow+\infty$. Moreover, if $\partial \Omega_{0}$ is smooth, then due to $u(x)=0$ for almost every $x \in \mathbb{R}^{N} \backslash \Omega_{0}$, we have $u \in H_{0}^{1}\left(\Omega_{0}\right)$. We claim that there exists $c_{0}>0$ independent of $\mu$ such that $\int_{\mathbb{R}^{N}}\left(\left|\nabla u_{\mu}\right|^{2}+u_{\mu}^{2}\right) d x \geq c_{0}$. Indeed, by $I_{\mu}^{\prime}\left(u_{\mu}\right)=0$, we have that

$$
0=\int_{\mathbb{R}^{N}}\left[\left|\nabla u_{\mu}\right|^{2}+(1+\mu g(x)) u_{\mu}^{2}\right] d x-\int_{\mathbb{R}^{N}} f\left(u_{\mu}\right) u_{\mu} d x .
$$

Then, by (2.3), there is $M>0$ independent of $\mu>0$ such that

$$
0 \geq \frac{1}{2} \int_{\mathbb{R}^{N}}\left(\left|\nabla u_{\mu}\right|^{2}+u_{\mu}^{2}\right) d x-M\left[\frac{1}{S} \int_{\mathbb{R}^{N}}\left(\left|\nabla u_{\mu}\right|^{2}+u_{\mu}^{2}\right) d x\right]^{2^{*} / 2} .
$$

Note that if

$$
\left[\int_{\mathbb{R}^{N}}\left(\left|\nabla u_{\mu}\right|^{2}+u_{\mu}^{2}\right) d x\right]^{\left(2^{*}-2\right) / 2}<\frac{S^{2^{*} / 2}}{4 M}
$$


then

$$
0 \geq \frac{1}{4} \int_{\mathbb{R}^{N}}\left(\left|\nabla u_{\mu}\right|^{2}+u_{\mu}^{2}\right) d x
$$

which implies that $u_{\mu}=0$, a contradiction. Thus, we obtain that

$$
\left[\int_{\mathbb{R}^{N}}\left(\left|\nabla u_{\mu}\right|^{2}+u_{\mu}^{2}\right) d x\right]^{\left(2^{*}-2\right) / 2} \geq \frac{S^{2^{*} / 2}}{4 M}
$$

and the claim is proved. Define the functional $L$ on $H_{0}^{1}\left(\Omega_{0}\right)$ by

$$
L(u)=\frac{1}{2} \int_{\Omega_{0}}\left(|\nabla u|^{2}+u^{2}\right) d x-\int_{\Omega_{0}} F(u) d x,
$$

where $u \in H_{0}^{1}\left(\Omega_{0}\right)$. Recall that $u_{\mu} \rightarrow u$ in $H^{1}\left(\mathbb{R}^{N}\right)$ as $\mu \rightarrow+\infty$. Then by $\int_{\mathbb{R}^{N}}\left(\left|\nabla u_{\mu}\right|^{2}+u_{\mu}^{2}\right) d x \geq c_{0}$, we have $u \neq 0$. On the other hand, due to $I_{\mu}^{\prime}\left(u_{\mu}\right)=0$, we have $L^{\prime}(u)=0$. A standard argument shows that $u$ is positive.

\section{Proof of Theorem 1.4}

In order to prove Theorem 1.4, we need the following abstract result established in [22].

Theorem 4.1. Let $X$ be a Banach space equipped with a norm $\|\cdot\|_{X}$ and let $J \subset \mathbb{R}^{+}$be an interval. We consider a family $\left(H_{\zeta}\right)_{\zeta \in J}$ of $\mathbb{C}^{1}$-functionals on $X$ having the form

$$
H_{\zeta}(u)=A(u)-\zeta B(u), \quad \text { for all } \zeta \in J,
$$

where $B(u) \geq 0$, for all $u \in X$, and either $A(u) \rightarrow+\infty$ or $B(u) \rightarrow+\infty$ as $\|u\|_{X} \rightarrow \infty$. We assume there are two points $v_{1}, v_{2}$ in $X$ such that

$$
c_{\zeta}=\inf _{\gamma \in \Gamma} \max _{t \in[0,1]} H_{\zeta}(\gamma(t))>\max \left\{H_{\zeta}\left(v_{1}\right), H_{\zeta}\left(v_{2}\right)\right\}, \quad \text { for all } \zeta \in J,
$$

where $\Gamma:=\left\{\gamma \in C([0,1], X): \gamma(0)=v_{1}, \gamma(1)=v_{2}\right\}$. Then, for almost every $\zeta \in J$, there is a sequence $\left\{v_{n}\right\} \subset X$ such that

(a) $\left\{v_{n}\right\}$ is bounded,

(b) $H_{\zeta}\left(v_{n}\right) \rightarrow c_{\zeta}$,

(c) $H_{\zeta}^{\prime}\left(v_{n}\right) \rightarrow 0$ in $X^{-1}$.

Moreover, the map $\zeta \rightarrow c_{\zeta}$ is continuous from the left.

Let $X:=\left\{u \in H^{1}\left(\mathbb{R}^{N}\right): u\right.$ is radial $\}$. For the simplicity, denote $\|\cdot\|_{H^{1}}=\|\cdot\|$. For $\lambda>((q-2) /(2 q))^{(q-2) / 2}\left(N S^{-N / 2}\right)^{(q-2) / 2} C_{q}^{q / 2}$ and $\zeta \in[1 / 2,1]$, define a family of functionals $H_{\zeta}^{\lambda}$ on $X$ by

$$
H_{\zeta}^{\lambda}(u)=\frac{1}{2}\|u\|^{2}-\zeta \int_{\mathbb{R}^{N}} F(u) d x .
$$

The principle of symmetric criticality implies that a critical point of $H_{\zeta}^{\lambda}$ on $X$ is a critical point of $H_{\zeta}^{\lambda}$ on $H^{1}\left(\mathbb{R}^{N}\right)$. Denote $H_{1}^{\lambda}(u)=H^{\lambda}(u)$. 
LEMma 4.2. For $\lambda>((q-2) /(2 q))^{(q-2) / 2}\left(N S^{-N / 2}\right)^{(q-2) / 2} C_{q}^{q / 2}$, there is $\gamma_{0} \in(0,1)$ such that for almost every $\zeta \in\left[1-\gamma_{0}, 1\right]$, there is a sequence $\left\{u_{n}^{\lambda}\right\} \subset X$ satisfying $\left\{u_{n}^{\lambda}\right\}$ is bounded, $H_{\zeta}^{\lambda}\left(u_{n}^{\lambda}\right) \rightarrow c_{\zeta}^{\lambda}$ and $\left(H_{\zeta}^{\lambda}\right)^{\prime}\left(u_{n}^{\lambda}\right) \rightarrow 0$. Moreover, $c_{\zeta}^{\lambda} \in\left(0, S^{N / 2} /\left(N \zeta^{(N-2) / 2}\right)\right)$ and the map $\zeta \rightarrow c_{\zeta}^{\lambda}$ is continuous from the left.

Proof. For $\lambda>((q-2) / 2 q)^{(q-2) / 2}\left(N S^{-N / 2}\right)^{(q-2) / 2} C_{q}^{q / 2}$, choose $\gamma_{0} \in(0,1)$ such that

$$
\max _{\zeta \in\left[1-\gamma_{0}, 1\right]} \zeta^{((N-2) / 2-2 /(q-2))}<\frac{2 q}{q-2} \frac{S^{N / 2}}{N} C_{q}^{-q /(q-2)} \lambda^{2 /(q-2)} .
$$

Set $J=\left[1-\gamma_{0}, 1\right], A(u)=\|u\|^{2} / 2$ and $B(u)=\int_{\mathbb{R}^{N}} F(x) d x$ in Theorem 4.1. It is easy to see that $B(u) \geq 0$ for all $u \in X$ and $A(u) \rightarrow+\infty$ as $\|u\| \rightarrow \infty$. Similarly to the proof of Lemma 2.1, we can prove that for almost every $\zeta \in\left[1-\gamma_{0}, 1\right]$, there is a bounded sequence $\left\{u_{n}^{\lambda}\right\} \subset X$ satisfying $H_{\zeta}^{\lambda}\left(u_{n}^{\lambda}\right) \rightarrow c_{\zeta}^{\lambda}$ and $\left(H_{\zeta}^{\lambda}\right)^{\prime}\left(u_{n}^{\lambda}\right) \rightarrow 0$. Moreover, the map $\zeta \mapsto c_{\zeta}^{\lambda}$ is continuous from the left. The definition of $c_{\zeta}^{\lambda}$ implies that $c_{\zeta}^{\lambda} \leq \sup _{t \geq 0} H_{\zeta}^{\lambda}(t \phi)$ with $\phi \in X$ satisfying

$$
C_{q}=\frac{\int_{\mathbb{R}^{N}}\left(|\nabla \phi|^{2}+|\phi|^{2}\right) d x}{\left(\int_{\mathbb{R}^{N}}|\phi|^{q} d x\right)^{2 / q}} .
$$

Then by $\left(f_{5}\right)$, we have

$$
\begin{array}{r}
\sup _{t \geq 0} H_{\zeta}^{\lambda}(t \phi) \leq \sup _{t \geq 0}\left[\frac{1}{2} t^{2} \int_{\mathbb{R}^{N}}\left(|\nabla \phi|^{2}+|\phi|^{2}\right) d x-\zeta \frac{\lambda}{q} t^{q} \int_{\mathbb{R}^{N}}|\phi|^{q} d x\right] \\
=\frac{1}{\zeta^{2 /(q-2)}}\left(\frac{1}{2}-\frac{1}{q}\right) \frac{1}{\lambda^{2 /(q-2)}} C_{q}^{q /(q-2)} .
\end{array}
$$

Together with (4.1), we get $c_{\zeta}^{\lambda}<S^{N / 2}\left(N \zeta^{(N-2) / 2}\right)$.

Lemma 4.3. For $\lambda>((q-2) /(2 q))^{(q-2) / 2}\left(N S^{-N / 2}\right)^{(q-2) / 2} C_{q}^{q / 2}$ and $\zeta \in$ $\left[1-\gamma_{0}, 1\right]$, let $\left\{u_{n}^{\lambda}\right\} \subset X$ be a sequence obtained in Lemma 4.2. Then $u_{n}^{\lambda} \rightarrow u_{\zeta}^{\lambda}$ in $X$.

Proof. Without loss of generality, we may assume that $u_{n}^{\lambda} \geq 0$ in $X$. Since $\left\|u_{n}^{\lambda}\right\|$ is bounded, we have $u_{n}^{\lambda} \rightarrow u_{\zeta}^{\lambda}$ weakly in $X$. Then $\left(H_{\zeta}^{\lambda}\right)^{\prime}\left(u_{\zeta}^{\lambda}\right)=0$. By $\left(\mathrm{f}_{1}\right)-\left(\mathrm{f}_{3}\right)$, we have

$$
\lim _{|t| \rightarrow \infty} \frac{h(t) t}{|t|^{2}+|t|^{2^{*}}}=0 \quad \text { and } \quad \lim _{t \rightarrow 0} \frac{h(t) t}{|t|^{2}+|t|^{2^{*}}}=0 .
$$

We also have that $\int_{\mathbb{R}^{N}}\left(\left|u_{n}^{\lambda}\right|^{2}+\left|u_{n}^{\lambda}\right|^{2^{*}}\right) d x$ is bounded. Then the compactness lemma of Strass [9], [33] implies that

$$
\lim _{n \rightarrow \infty} \int_{\mathbb{R}^{N}}\left|h\left(u_{n}^{\lambda}\right) u_{n}^{\lambda}-h\left(u_{\zeta}^{\lambda}\right) u_{\zeta}^{\lambda}\right| d x=0 .
$$


Similarly to (4.3), there holds

$$
\lim _{n \rightarrow \infty} \int_{\mathbb{R}^{N}} H\left(u_{n}^{\lambda}\right) d x=\int_{\mathbb{R}^{N}} H\left(u_{\zeta}^{\lambda}\right) d x .
$$

Set $v_{n}^{\lambda}=u_{n}^{\lambda}-u_{\zeta}^{\lambda}$. Then by (4.4) and the Brezis-Lieb lemma in [37],

$$
c_{\zeta}^{\lambda}-H_{\zeta}^{\lambda}\left(u_{\zeta}^{\lambda}\right)=\frac{1}{2}\left\|v_{n}^{\lambda}\right\|^{2}-\frac{\zeta}{2^{*}} \int_{\mathbb{R}^{N}}\left|v_{n}^{\lambda}\right|^{2^{*}} d x+o_{n}(1) .
$$

On the other hand, by (4.3) and the Brezis-Lieb lemma,

$$
o_{n}(1)=\left(\left(H_{\zeta}^{\lambda}\right)^{\prime}\left(u_{n}^{\lambda}\right), u_{n}^{\lambda}\right)-\left(\left(H_{\zeta}^{\lambda}\right)^{\prime}\left(u_{\zeta}^{\lambda}\right), u_{\zeta}^{\lambda}\right)=\left\|v_{n}^{\lambda}\right\|^{2}-\zeta \int_{\mathbb{R}^{N}}\left|v_{n}^{\lambda}\right|^{2^{*}} d x
$$

Note that $\left(H_{\zeta}^{\lambda}\right)^{\prime}\left(u_{\zeta}^{\lambda}\right)=0$. Similarly to Proposition 1 in [9], we have the Pohožaev type identity:

$$
\frac{N-2}{2 N} \int_{\mathbb{R}^{N}}\left|\nabla u_{\zeta}^{\lambda}\right|^{2} d x=\int_{\mathbb{R}^{N}}\left[\zeta F\left(u_{\zeta}^{\lambda}\right)-\frac{1}{2}\left|u_{\zeta}^{\lambda}\right|^{2}\right] d x .
$$

Then, by (4.7), there holds

$$
H_{\zeta}^{\lambda}\left(u_{\zeta}^{\lambda}\right)=\frac{1}{N} \int_{\mathbb{R}^{N}}\left|\nabla u_{\zeta}^{\lambda}\right|^{2} d x \geq 0
$$

from which we have $c_{\zeta}^{\lambda}-H_{\zeta}^{\lambda}\left(u_{\zeta}^{\lambda}\right) \leq c_{\zeta}^{\lambda}<S^{N / 2} /\left(N \zeta^{(N-2) / 2}\right)$. Assume that $\left\|v_{n}^{\lambda}\right\|^{2} \rightarrow l \geq 0$. By (4.6), we get that $\zeta \int_{\mathbb{R}^{N}}\left|v_{n}^{\lambda}\right|^{2^{*}} d x \rightarrow l$. We claim that $l=0$. Otherwise, we have $l>0$. The Sobolev embedding theorem implies that

$$
S \leq \frac{\left\|v_{n}^{\lambda}\right\|^{2}}{\left(\int_{\mathbb{R}^{N}}\left|v_{n}^{\lambda}\right|^{2^{*}} d x\right)^{2 / 2^{*}},}
$$

hence we have $l \geq S^{N / 2} / \zeta^{(N-2) / 2}$. Thus, by $(4.5)$, there holds $c_{\zeta}^{\lambda}-H_{\zeta}^{\lambda}\left(u_{\zeta}^{\lambda}\right)=$ $l / N \geq S^{N / 2} /\left(N \zeta^{(N-2) / 2}\right)$, a contradiction with $c_{\zeta}^{\lambda}-H_{\zeta}^{\lambda}\left(u_{\zeta}^{\lambda}\right)<S^{N / 2} /\left(N \zeta^{(N-2) / 2}\right)$. Then we have $l=0$, which implies that $u_{n}^{\lambda} \rightarrow u_{\zeta}^{\lambda}$ in $X$.

Proof of Theorem 1.4. By Lemmas 4.2 and 4.3, for almost every $\zeta \in[1-$ $\left.\gamma_{0}, 1\right]$, we have $H_{\zeta}^{\lambda}\left(u_{\zeta}^{\lambda}\right)=c_{\zeta}^{\lambda} \in\left(0, S^{N / 2} /\left(N \zeta^{(N-2) / 2}\right)\right)$ and $\left(H_{\zeta}^{\lambda}\right)^{\prime}\left(u_{\zeta}^{\lambda}\right)=0$. The maximum principle implies that $u_{\zeta}^{\lambda}$ is positive. Choose $\zeta_{n} \in\left[1-\gamma_{0}, 1\right]$ such that $\zeta_{n} \rightarrow 1,\left(H_{\zeta_{n}}^{\lambda}\right)^{\prime}\left(u_{\zeta_{n}}^{\lambda}\right)=0$ and $H_{\zeta_{n}}^{\lambda}\left(u_{\zeta_{n}}^{\lambda}\right)=c_{\zeta_{n}}^{\lambda} \in\left(0, S^{N / 2} /\left(N \zeta_{n}^{(N-2) / 2}\right)\right)$. Then, by (4.7), we obtain that $c_{\zeta_{n}}^{\lambda}=(1 / N) \int_{\mathbb{R}^{N}}\left|\nabla u_{\zeta_{n}}^{\lambda}\right|^{2} d x$ is bounded. The Sobolev embedding theorem implies that $\int_{\mathbb{R}^{N}}\left|u_{\zeta_{n}}^{\lambda}\right|^{2^{*}} d x$ is bounded. From (2.3) and $\left(\left(H_{\zeta_{n}}^{\lambda}\right)^{\prime}\left(u_{\zeta_{n}}^{\lambda}\right), u_{\zeta_{n}}^{\lambda}\right)=0$, we can derive that $\left\|u_{\zeta_{n}}^{\lambda}\right\|$ is bounded. On the other hand, due to boundedness of $\left\|u_{\zeta_{n}}^{\lambda}\right\|, H^{\lambda}\left(u_{\zeta_{n}}^{\lambda}\right)=H_{\zeta_{n}}^{\lambda}\left(u_{\zeta_{n}}^{\lambda}\right)+\left(\zeta_{n}-1\right) \int_{\mathbb{R}^{N}} F\left(u_{\zeta_{n}}^{\lambda}\right) d x$ and $\lim _{n \rightarrow \infty} c_{\zeta_{n}}^{\lambda}=c_{1}^{\lambda} \in\left(0, S^{N / 2} / N\right)$, we obtain that $\lim _{n \rightarrow \infty} H^{\lambda}\left(u_{\zeta_{n}}^{\lambda}\right)=c_{1}^{\lambda} \in\left(0, S^{N / 2} / N\right)$ and $\lim _{n \rightarrow \infty}\left(H^{\lambda}\right)^{\prime}\left(u_{\zeta_{n}}^{\lambda}\right)=0$. 
Assume that $u_{\zeta_{n}}^{\lambda} \rightarrow u_{0}^{\lambda}$ weakly in $X$. Then $\left(H^{\lambda}\right)^{\prime}\left(u_{0}^{\lambda}\right)=0$. We claim that $u_{0}^{\lambda} \neq 0$. Otherwise, we have $u_{\zeta_{n}} \rightarrow 0$ weakly in $X$, from which we get $u_{\zeta_{n}}^{\lambda} \rightarrow 0$ in $L^{t}\left(\mathbb{R}^{N}\right)$, for all $t \in\left(2,2^{*}\right)$. Then, by $\left(\mathrm{f}_{1}\right)-\left(\mathrm{f}_{3}\right)$, we derive that

$$
\int_{\mathbb{R}^{N}} H\left(u_{\zeta_{n}}^{\lambda}\right) d x=o_{n}(1) \quad \text { and } \quad \int_{\mathbb{R}^{N}} h\left(u_{\zeta_{n}}^{\lambda}\right) u_{\zeta_{n}}^{\lambda} d x=o_{n}(1) .
$$

Note that $\lim _{n \rightarrow \infty} H^{\lambda}\left(u_{\zeta_{n}}^{\lambda}\right)=c_{1}^{\lambda}$ and $\lim _{n \rightarrow \infty}\left(H^{\lambda}\right)^{\prime}\left(u_{\zeta_{n}}^{\lambda}\right)=0$. We have

$\frac{1}{2}\left\|u_{\zeta_{n}}^{\lambda}\right\|^{2}-\frac{1}{2^{*}} \int_{\mathbb{R}^{N}}\left|u_{\zeta_{n}}^{\lambda}\right|^{2^{*}} d x=c_{1}^{\lambda}+o_{n}(1) \quad$ and $\quad\left\|u_{\zeta_{n}}^{\lambda}\right\|^{2}-\int_{\mathbb{R}^{N}}\left|u_{\zeta_{n}}^{\lambda}\right|^{2^{*}} d x=o_{n}(1)$.

Similarly to the proof of Lemma 4.3, we can derive that $u_{\zeta_{n}}^{\lambda} \rightarrow 0$, a contradiction with $c_{1}^{\lambda}>0$. Thus, we have $u_{0}^{\lambda} \neq 0$. The maximum principle implies that $u_{0}^{\lambda}$ is positive.

Now we claim that $c_{1}^{\lambda} \geq H^{\lambda}\left(u_{0}^{\lambda}\right)$. In fact, from equations $\left(H_{\zeta_{n}}^{\lambda}\right)^{\prime}\left(u_{\zeta_{n}}^{\lambda}\right)=0$, $\left(H^{\lambda}\right)^{\prime}\left(u_{0}^{\lambda}\right)=0$ and (4.7), we have

$$
c_{\zeta_{n}}^{\lambda}=H_{\zeta_{n}}^{\lambda}\left(u_{\zeta_{n}}^{\lambda}\right)=\frac{1}{N} \int_{\mathbb{R}^{N}}\left|\nabla u_{\zeta_{n}}^{\lambda}\right|^{2} d x \quad \text { and } \quad H^{\lambda}\left(u_{0}^{\lambda}\right)=\frac{1}{N} \int_{\mathbb{R}^{N}}\left|\nabla u_{0}^{\lambda}\right|^{2} d x .
$$

Then by the Fatou lemma, we derive that

$$
c_{1}^{\lambda}=\lim _{n \rightarrow \infty} c_{\zeta_{n}}^{\lambda} \geq \frac{1}{N} \int_{\mathbb{R}^{N}}\left|\nabla u_{0}^{\lambda}\right|^{2} d x=H^{\lambda}\left(u_{0}^{\lambda}\right) .
$$

Let $m^{\lambda}:=\inf \left\{H^{\lambda}(u): u \in H^{1}\left(\mathbb{R}^{N}\right), u>0,\left(H^{\lambda}\right)^{\prime}(u)=0\right.$ in $\left.H^{-1}\left(\mathbb{R}^{N}\right)\right\}$. The principle of symmetric criticality implies that $\left(H^{\lambda}\right)^{\prime}\left(u_{0}^{\lambda}\right)=0$ in $H^{-1}\left(\mathbb{R}^{N}\right)$. Then we have $m^{\lambda} \leq H^{\lambda}\left(u_{0}^{\lambda}\right) \leq c_{1}^{\lambda}<S^{N / 2} / N$. By the definition of $m^{\lambda}$, there exists $\left\{u_{n}^{\lambda}\right\} \subset H^{1}\left(\mathbb{R}^{N}\right)$ such that $u_{n}^{\lambda}>0, H^{\lambda}\left(u_{n}^{\lambda}\right) \rightarrow m^{\lambda}$ and $\left(H^{\lambda}\right)^{\prime}\left(u_{n}^{\lambda}\right)=0$. Similarly to the proof that $\left\|u_{\zeta_{n}}^{\lambda}\right\|$ is bounded, we can derive that $\left\|u_{n}^{\lambda}\right\|$ is bounded. On the other hand, due to $(2.3)$ and $\left(\left(H^{\lambda}\right)^{\prime}\left(u_{n}^{\lambda}\right), u_{n}^{\lambda}\right)=0$, we have for all $\varepsilon>0$ that there exists $C(\varepsilon)>0$ such that

$$
\left\|u_{n}^{\lambda}\right\|^{2} \leq \varepsilon \int_{\mathbb{R}^{N}}\left|u_{n}^{\lambda}\right|^{2} d x+C(\varepsilon) \int_{\mathbb{R}^{N}}\left|u_{n}^{\lambda}\right|^{2^{*}} d x .
$$

Choose $\varepsilon>0$ small enough. From the Sobolev embedding theorem, there holds

$$
\int_{\mathbb{R}^{N}}\left|\nabla u_{n}^{\lambda}\right|^{2} d x \leq C\left(\int_{\mathbb{R}^{N}}\left|\nabla u_{n}^{\lambda}\right|^{2} d x\right)^{2^{*} / 2} .
$$

Thus, there exists $\varrho>0$ independent of $n$ such that

$$
\int_{\mathbb{R}^{N}}\left|\nabla u_{n}^{\lambda}\right|^{2} d x \geq \varrho .
$$

Together with $\left(H^{\lambda}\right)^{\prime}\left(u_{n}^{\lambda}\right)=0$ and (4.7), we have

$$
m^{\lambda}+o_{n}(1)=\frac{1}{N} \int_{\mathbb{R}^{N}}\left|\nabla u_{n}^{\lambda}\right|^{2} d x \geq \frac{1}{N} \varrho,
$$

which implies that $m^{\lambda}>0$. 
Now we claim that there exists $\left\{y_{n}^{\lambda}\right\} \subset \mathbb{R}^{N}$ such that $u_{n}^{\lambda}\left(\cdot+y_{n}^{\lambda}\right) \rightarrow u^{\lambda} \neq 0$ weakly in $H^{1}\left(\mathbb{R}^{N}\right)$. If $u_{n}^{\lambda} \rightarrow u^{\lambda} \neq 0$ weakly in $H^{1}\left(\mathbb{R}^{N}\right)$, it is obvious that the claim is true, so we may assume that $u_{n}^{\lambda} \rightarrow 0$ weakly in $H^{1}\left(\mathbb{R}^{N}\right)$. Then either

$$
\lim _{n \rightarrow \infty} \sup _{y \in \mathbb{R}^{N}} \int_{B_{1}(y)}\left|u_{n}^{\lambda}\right|^{2} d x=0
$$

or there exists $\nu>0$ such that

$$
\lim _{n \rightarrow \infty} \sup _{y \in \mathbb{R}^{N}} \int_{B_{1}(y)}\left|u_{n}^{\lambda}\right|^{2} d x \geq \nu>0 .
$$

If (4.8) holds, by the Lions lemma in [37], we get $u_{n}^{\lambda} \rightarrow 0$ in $L^{t}\left(\mathbb{R}^{N}\right)$ for all $t \in\left(2,2^{*}\right)$. Together with $H^{\lambda}\left(u_{n}^{\lambda}\right) \rightarrow m^{\lambda}$ and $\left(\left(H^{\lambda}\right)^{\prime}\left(u_{n}^{\lambda}\right), u_{n}^{\lambda}\right)=0$, there holds

$\frac{1}{2}\left\|u_{n}^{\lambda}\right\|^{2}-\frac{1}{2^{*}} \int_{\mathbb{R}^{N}}\left|u_{n}^{\lambda}\right|^{2^{*}} d x=m^{\lambda}+o_{n}(1) \quad$ and $\quad\left\|u_{n}^{\lambda}\right\|^{2}-\int_{\mathbb{R}^{N}}\left|u_{n}^{\lambda}\right|^{2^{*}} d x=o_{n}(1)$.

Similarly to the proof of Lemma 4.2 , we can derive that $u_{n}^{\lambda} \rightarrow 0$ in $H^{1}\left(\mathbb{R}^{N}\right)$, a contradiction with $m^{\lambda}>0$. Then we have (4.9). Thus, there exists $\left\{y_{n}^{\lambda}\right\} \subset \mathbb{R}^{N}$ such that $u_{n}^{\lambda}\left(\cdot+y_{n}^{\lambda}\right) \rightarrow u^{\lambda} \neq 0$ weakly in $H^{1}\left(\mathbb{R}^{N}\right)$. Together with $H^{\lambda}\left(u_{n}^{\lambda}\right) \rightarrow m^{\lambda}$ and $\left(H^{\lambda}\right)^{\prime}\left(u_{n}^{\lambda}\right)=0$, we know $u^{\lambda}$ is positive, $\left(H^{\lambda}\right)^{\prime}\left(u^{\lambda}\right)=0$ and $m^{\lambda} \geq H^{\lambda}\left(u^{\lambda}\right)$. Since $\left(H^{\lambda}\right)^{\prime}\left(u^{\lambda}\right)=0$, we also have $m^{\lambda} \leq H^{\lambda}\left(u^{\lambda}\right)$ from the definition of $m^{\lambda}$. Then $H^{\lambda}\left(u^{\lambda}\right)=m^{\lambda}$. Thus, problem (1.1) admits a positive ground state solution $u^{\lambda}$ for $\mu=0$.

On the other hand, we know $m^{\lambda} \leq c_{1}^{\lambda}$. The definition of $c_{1}^{\lambda}$ implies that $c_{1}^{\lambda} \leq \sup _{t \geq 0} H^{\lambda}(t \phi)$ with $\phi \in H^{1}\left(\mathbb{R}^{N}\right)$ satisfying

$$
C_{q}=\frac{\int_{\mathbb{R}^{N}}\left(|\nabla \phi|^{2}+|\phi|^{2}\right) d x}{\left(\int_{\mathbb{R}^{N}}|\phi|^{q} d x\right)^{2 / q} .}
$$

Then, by (4.2), we have $\lim _{\lambda \rightarrow \infty} m^{\lambda} \leq \lim _{\lambda \rightarrow \infty} c_{1}^{\lambda} \leq \lim _{\lambda \rightarrow \infty} \sup _{t \geq 0} H^{\lambda}(t \phi)=0$.

Acknowledgements. The authors are grateful to the anonymous referee for valuable comments and suggestions.

\section{REFERENCES}

[1] C.O. Alves, M.A.S. Souto And M. Montenegro, Existence of a ground state solution for a nonlinear scalar field equation with critical growth, Calc. Var. Partial Differential Equations 43 (2012), 537-554.

[2] A. Ambrosetti And P.H. Rabinowitz, Dual variational methods in critical point theory and applications, J. Funct. Anal. 14 (1973), 349-381.

[3] A. Ambrosetti, M. Badiale and S. Cingolani, Semiclassical states of nonlinear Schrödinger equations, Arch. Ration. Mech. Anal. 140 (1997), 285-300.

[4] _ Multiplicity results for some nonlinear Schrödinger equations with potentials, Arch. Ration. Mech. Anal. 159 (2001), 253-271. 
[5] T. BARtsch, A. PANkov AND Z.-Q. WANG, Nonlinear Schrödinger equations with steep potential well, Commun. Contemp. Math. 3 (2001), 549-569.

[6] T. BARTSCH AND Z.-Q. WANG, Existence and multiplicity results for some superlinear elliptic problems on $\mathbb{R}^{N}$, Comm. Partial Differential Equations 20(1995), 1725-1741.

[7] _ Multiple positive solutions for a nonlinear Schrödinger equation, Z. Angew. Math. Phys. 51 (2000), 366-384.

[8] H. Berestycki, T. Gallouët and O. Kavian, Equations de champs scalaires euclidiens non linéaire dans le plan, C.R. Acad. Sci. Paris Paris Ser. I Math. 297 (1983), 307-310.

[9] H. Berestycki And P.L. Lions, Nonlinear scalar field equations I. Existence of a ground state, Arch. Ration. Mech. Anal. 82 (1983), 313-346.

[10] J. BYEON AND L. JEANJEAN, Standing waves for nonlinear Schrödinger equations with a general nonlinearity, Arch. Ration. Mech. Anal. 185 (2007), 185-200.

[11] G. Cerami, Some nonlinear elliptic problems in unbounded domains, Milan J. Math. 74 (2006), 47-77.

[12] M. Clapp AND Y.H. Ding, Positive solutions of a Schrödinger equation with critical nonlinearity, Z. Angew. Math. Phys. 55 (2004), 592-605.

[13] S. Cingolani And A. Pistoia, Nonexistence of single blow-up solutions for a nonlinear Schrödinger equation involving critical Sobolev exponent, Z. Angew. Math. Phys. 55 (2004), 201-215.

[14] D.G. De Figueiredo And Y.H. Ding, Solutions of a nonlinear Schrödinger equation, Discrete Contin. Dynam. Sys. 8 (2002), 563-584.

[15] M. DEL Pino AND P. FElmer, Local mountain pass for semilinear elliptic problems in unbounded domains, Calc. Var. Partial Differential Equations 4 (1996), 121-137.

[16] _ Multi-peak bound states for nonlinear Schrodinger equations, Ann. Inst. H. Poincaré Anal. Nonlinéaire 15 (1998), 127-149.

[17] _ Semiclassical states for nonlinear Schrodinger equations, J. Funct. Anal. 149 (1997), 245-265.

[18] _ Semi-classical states of nonlinear Schrodinger equations: a variational reduction method, Math. Ann. 324 (2002), 1-32.

[19] Y.H. Ding AND K. TANAKA, Multiplicity of positive solutions of a nonlinear Schrodinger equation, Manuscripta Math. 112 (2003), 109-135.

[20] A. Floer And A. Weinstein, Nonspreading wave packets for the cubic Schrödinger equations with a bounded potential, J. Funct. Anal. 69 (1986), 397-408.

[21] C. GuI, Existence of multi-bump solutions for nonlinear Schrödinger equations via variational methods, Comm. Partial Differential Equations 21 (1996), 787-820.

[22] L. JeAnJeAn, On the existence of bounded Palais-Smale sequences and application to a Landesman-Lazer-type problem set on $\mathbb{R}^{N}$, Proc. Roy. Soc. Edinburgh 129 (1999), 787-809.

[23] L. JEANJEAN AND K. TANAKA, A positive solution for asymptotically linear elliptic problem on $\mathbb{R}^{N}$ autonomous at infinity, ESAIM Control Optim. Calc. Var. 7 (2002), 597-614.

[24] _ A positive solution for a nonlinear Schrödinger equation on $\mathbb{R}^{N}$, Indiana Univ. Math. J. 54 (2005), 443-464.

[25] _ Singularly perturbed elliptic problems with superlinear or asymptotically linear nonlinearities, Calc. Var. Partial Differential Equations 21 (2004), 287-318.

[26] Y.S. JiAng AND H.S. Zhou, Schrödinger-Poisson system with steep potential well, J. Differential Equations 251 (2011), 582-608.

[27] Y.Q. Li, Z.-Q. WANG AND J. ZENG, Ground states of nonlinear Schrödinger equations with potentials, Ann. Inst. H. Poincaré Anal. Non-linéaire 23 (2006), 829-837. 
[28] Y.Y. LI, On a singularly perturbed elliptic equation, Adv. Differential Equations 2 (1997), 955-980.

[29] Y.-G. ОH, Existence of semi-classical bound states of nonlinear Schrodinger equation with potential on the class $(V)_{a}$, Comm. Partial Differential Equations 13 (1988), 1499-1519.

[30] _ On positive multi-lump bound states of nonlinear Schrodinger equations under multiple well potential, Comm. Math. Phys. 131 (1990), 223-253.

[31] P.H. Rabinowitz, On a class of nonlinear Schrödinger equations, Z. Angew. Math. Phys. 43 (1992), 270-291.

[32] Y. Sato AND K. TANAKa, Sign-changing multi-bump solutions for nonlinear Schrödinger equations with steep potential wells, Transactions of the American Mathematical Society 361 (2009), 6205-6253.

[33] W.A. Strauss, Existence of solitary waves in higher dimensions, Comm. Math. Phys. 55 (1977), 149-162.

[34] C.A. StuART And H.S. Zhou, Global branch of solutions for non-linear Schrodinger equations with deepening potential well, Proc. London Math. Soc. 92 (2006), 655-681.

[35] F.A. VAn HeERden, Multiple solutions for a Schrödinger type equation with an asymptotically linear term, Nonlinear Anal. 55 (2003), 739-758.

[36] F.A. VAN HEERDEN AND Z.-Q. WANG, Schrödinger type equations with asymptotically linear nonlinearities, Differential Integral Equations 16 (2003), 257-280.

[37] M. Willem, Minimax Theorems, Birkhäuser, Boston (1996).

[38] J.J. Zhang, Z. Chen And W. Zou, Standing waves for nonlinear Schrödinger equations involving critical growth, submitted.

[39] J. Zhang And W. Zou, The critical case for a Berestycki-Lions theorem, Sci. China Math. 57 (2014), 541-555.

[40] _ , A Berestycki-Lions theorem revisited, Comm. Contemp. Math. 14 (2012), 1250033.

JiAN ZHANG

College of Science

China University of Petroleum

Qingdao 266555, Shandong, P.R. CHINA

E-mail address: zjianmath@163.com

Wenming Zou

Department of Mathematical Sciences

Tsinghua University

Beijing 100084, P.R. CHINA

E-mail address: wzou@math.tsinghua.edu.cn 